\title{
Identification of an autophagy-related 10-lncRNA-mRNA signature for distinguishing glioblastoma multiforme from lower-grade glioma and prognosis prediction
}

\author{
Bo Wei ${ }^{1, \star}$, Le Wang ${ }^{2, \star}$ and Jingwei Zhao ${ }^{1}$ \\ ${ }^{1}$ Department of Neurosurgery, China-Japan Union Hospital of Jilin University, Changchun, Jilin, China \\ ${ }^{2}$ Department of Ophthalmology, The First Hospital of Jilin University, Changchun, Jilin, China
}

\begin{abstract}
Autophagy may provide the source of nutrients for tumor cells. We aim to develop an autophagy-related signature to predict the progression from lower-grade gliomas (LGG) to glioblastoma multiforme (GBM) and prognosis. Totally, 686 differentially expressed genes (DEGs) and 73 long non-coding RNAs (DELs) were identified between GBM and LGG samples from the Chinese Glioma Genome Atlas (CGGA). Of them, 131 DEGs were intersected with autophagy genes from the Human Autophagy Database; while 54 DELs co-expressed with autophagy-related DEGs. Ten autophagy-related genes were associated with overall survival and could distinguish GBM from LGG, with the accuracy of 0.891 using CGGA dataset and 0.790 using The Cancer Genome Atlas (TCGA) dataset. The risk score was established based on these 10 genes. Patients with higher risk score were at an increased risk of developing GBM (49.7\% vs. $21.3 \%$; $p<0.001)$ and worse prognosis than those in low risk group. The prognostic accuracy was 0.840 and 0.744 for CGGA and TCGA dataset, respectively. Age, recurrence, isocitrate dehydrogenase mutation and risk score were independent prognostic factors and thus they were used to build a nomogram which showed the highest prognostic power. This established nomogram may aid the clinical decision making of personalized treatment.
\end{abstract}

Key words: Glioblastoma - Lower-grade glioma - Nomogram - Prognostic signature Autophagy-related genes - Autophagy-related lncRNAs

\section{Introduction}

Glioma is the most common category of primary central nervous system tumors (Almutrafi et al. 2020; Araghi et al. 2020), accounting for approximately $35 \%$ of all burdens. Despite huge advance has been made in the diagnosis and treatment of glioma, the overall mortality is still high, which is especially obvious in the patients with high-grade gliomas (glioblastoma, GBM; 95\%) relative to lower-grade gliomas (LGG, 50\%) because of its aggressive and invasive nature

Electronic supplementary material. The online version of this article (doi: 10.4149/gpb_2021008) contains Supplementary material.

\footnotetext{
* These authors contributed to this work equally.

Correspondence to: Jingwei Zhao, Departments of Neurosurgery, China-Japan Union Hospital of Jilin University, No. 126 Xiantai Street, Changchun, Jilin 130033, China

E-mail: jwzhao@jlu.edu.cn
}

(Ostrom et al. 2018). Therefore, it is necessary to early stratify the patients at a high risk of developing GBM to schedule individualized treatment and improve survival.

With the development of molecular biology and bioinformatics, recent studies indicated there were obvious differences in gene expression profile between GBM and LGG (Wang and Ma 2019; Wang et al. 2019a, 2019b; Wu et al. 2019; Zhang et al. 2019, 2020; Biterge-Sut 2020). Thus, identification of molecular biomarkers may be underlying approaches to distinguish GBM from LGG and predict the prognosis. This hypothesis has been demonstrated by several authors. For example, $\mathrm{Wu}$ et al. (2019) found lipid metabolism-related genes were differentially expressed between GBM and LGG. Nine of them were identified to serve as a classifier for stratifying different grades. Also, this nine-gene established risk score showed strong prognostic power for glioma patients [the area under the receiver operating characteristic (ROC) curve (AUC) $=$ 0.86 for The Cancer Genome Atlas (TCGA) dataset; AUC = 
0.82 for the Chinese Glioma Genome Atlas (CGGA) dataset (Wu et al. 2019). Wang et al. (2019a) observed the distribution of pseudogenes was significantly different between GBM and LGG samples. Five of them were shown to be associated with overall survival (OS) and used to construct the risk score. GBM had significantly higher risk scores than LGG and may be more frequently assigned into the high risk score to result in poor OS. The predictive accuracy of this risk score for 5-year OS in glioma patients was 0.876 and 0.912 using the training and validation dataset, respectively. The study of Wang and $\mathrm{Ma}$ (2019) revealed the gene expression patterns of necrosis-related genes were significantly different between GBM and LGG. Seven genes were correlated with the outcome of GBM/LGG patients and integrated to generate the risk score. Survival analysis suggested that GBM/LGG patients in the high-risk group had worse OS than those in the low-risk. However, the predictors for stratifying GBM and LGG and their prognosis remain rarely reported.

Autophagy is a cellular process responsible for lysosomal degradation of damaged, denatured or aging proteins and organelles. In normal cells, the activation of autophagy protects cells against toxic injury and stress, and hereby dampens malignant transformation (Li et al. 2020). However, in tumor cells, the recycled proteins and organelles produced by autophagy may be the source of nutrients and energy for tumor growth, invasion and metastasis (Yang et al. 2019; Li et al. 2020). Theoretically, autophagy-related genes may represent promising biomarkers to predict the tumor progression (such as from LGG to GBM) and poor prognosis. This speculation has been verified in some studies. Jiang and colegue detected the expression levels of autophagic protein light chain 3 (LC3) B and p62 were higher in GBM than those in LGG tissues. High levels of LC3B and $\mathrm{p} 62$ protein were also associated with advanced tumor stages, worse relapse-free survival (RFS) and OS in glioma patients (Jiang and Wu 2018). The results of Padmakrishnan et al. (2019) showed autophagy proteins (LC3 and beclin 1) were highly expressed in GBM compared with LGG. Patients with low LC3/beclin 1 expression had better progression free survival (PFS) than those with high expression of LC3/beclin 1. There were also several studies to investigate a prognostic signature constituted by autophagy-related mRNAs (Wang et al. 2019c; 2020a; 2020b; 2021) for glioma patients. Nevertheless, they either focused on the genes differentially expressed only between glioma and normal controls (Wang et al. 2019c, Wang et al. 2021) or did not perform the differential analysis (Wang et al. 2020b; Wang et al. 2020c). No studies used the genes differentially expressed between GBM and LGG. In addition, Luan et al. (2019) suggested long non-coding RNAs (lncRNAs) that could co-express with autophagy-related genes also had prognostic potential for glioma, but in which lncRNAs and mRNAs were also not differentially expressed.
In present study, we aimed to develop a new autophagyrelated signature based on the differentially expressed genes (DEGs) and lncRNAs (DELs) between GBM and LGG. This signature may effectively diagnose GBM patients from LGG patients and predict the poor prognostic outcomes for highrisk populations (including GBM and possibly progress to GBM).

\section{Materials and Methods}

\section{Data source}

The RNA-seq expression data (fragments mapped per kilobase of exon per million reads mapped, level 3) and clinical information were collected from CGGA (http://www.cgga.org.cn) and TCGA (https://portal.gdc.cancer.gov) public databases. CGGA dataset (including 431 LGG and 237 GBM samples) was used as the training set; while TCGA dataset (containing 520 LGG and 152 GBM samples) was set as the testing set (ID of each sample is shown in Supplementary material, Table S1).

\section{Identification of autophagy-related DEGs and DELs}

The mRNAs and lncRNAs in CGGA and TCGA datasets were annotated by the HUGO Gene Nomenclature Committee (HGNC; http://www.genenames.org/) that assigns a unique symbol and name to 4,516 lncRNAs and 19,200 protein-coding genes (Povey et al. 2001). DEGs and DELs were identified between GBM and LGG using package limma of R (version 3.34.7; https://bioconductor.org/packages/release/bioc/html/ limma.html) (Ritchie et al. 2015). False discovery rate (FDR) $<0.05$ and $\mid \log _{2} \mathrm{FC}$ (fold change) $\mid>1$ were set as the statistical threshold. Bidirectional hierarchical clustering was performed based on all differentially expressed RNAs (DERs) using package pheatmap of R (version: 1.0.8; https://cran.r-project.org/ web/packages/pheatmap). The autophagy-related gene lists were downloaded from the Human Autophagy Database (HADb, http://www.autophagy.lu/), which were then compared with the DEGs to screen the overlap (that is, autophagy-related DEGs). Pearson correlation coefficients (PCC) were calculated by cor.test function (https://stat.ethz.ch/R-manual/R-devel/ library/stats/html/cor.test.html) to explore the correlation between the DELs and autophagy-related DEGs. DELs with a PCC $>0.4$ were defined as autophagy-related lncRNAs. The co-expression network was established based on the interaction pairs between DELs and DEGs and visualized using Cytoscape software (version 3.6.1; www.cytoscape.org/).

\section{Function enrichment analysis}

The functions of autophagy-related DEGs in the coexpression network were analyzed using the Database for 
Annotation, Visualization and Integrated Discovery (DAVID) (version 6.8; http://david.abcc.ncifcrf.gov) (Dennis et al. 2003). Gene Ontology (GO) biological process terms and Kyoto Encyclopedia of Genes and Genomes (KEGG) pathways were enriched. FDR $<0.05$ was considered to be statistically significant.

\section{Development of prognostic signature}

Based on the clinical prognosis information in 668 samples of CGGA, univariate Cox regression analysis was used to filter the DELs and DEGs of the co-expression network that were significantly associated with the OS. The DELs and DEGs with a log-rank $p<0.05$ in univariate analysis were entered into the multivariate Cox regression model for identifying independent prognostic genes. Logit regression model in glm function of $\mathrm{R}$ was further utilized on these independent prognostic genes to identify the feature genes that could effectively distinguish
GBM from LGG. The risk score model was established for each patient by combining the expression of the feature prognostic signature and their prognostic coefficients in multivariate analysis.

The patients were divided into the high-risk group and the low-risk group by selecting the median risk score as the cut-off. The prognostic differences between the two groups were analyzed by plotting Kaplan-Meier survival curve and performing log-rank test. The predictive accuracy of the autophagy-related signature was assessed by drawing ROC curve and calculating AUC. These analyses were carried out for the training dataset (CGGA) and testing dataset (TCGA), respectively.

To explore whether the risk score was independent of clinicopathological factors, univariate and multivariate Cox regression analyses were performed using the CGGA cohort. Stratification analysis was subsequently applied for clinical variables with $p<0.05$ in multivariate analysis to further evaluate the prognostic significance of risk score. A nomogram comprising all the variables significant in the
A

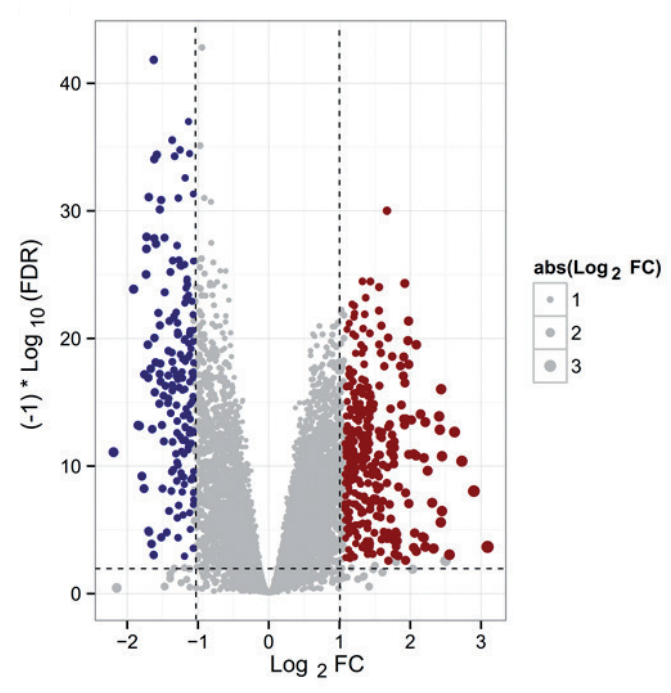

B

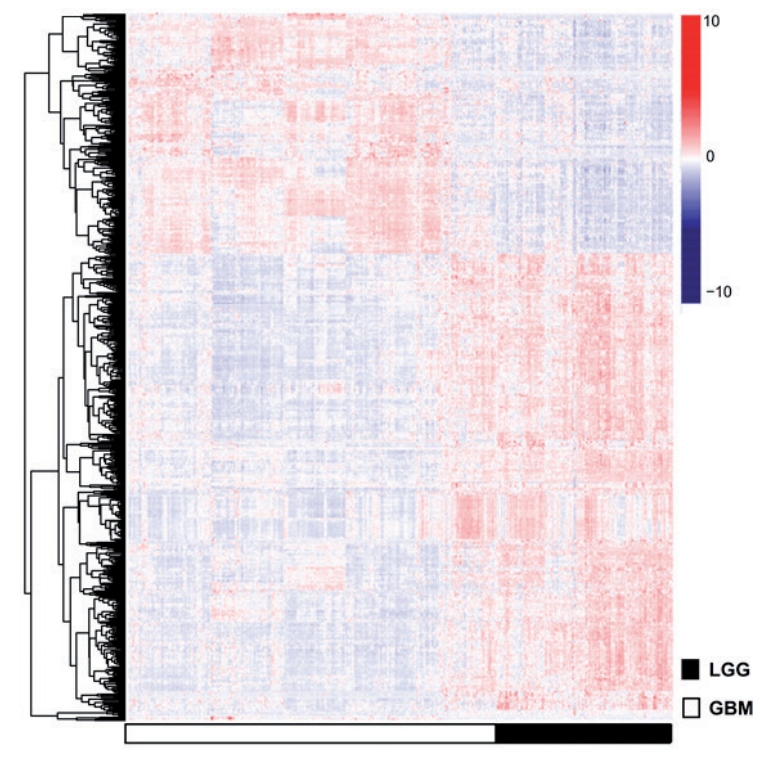

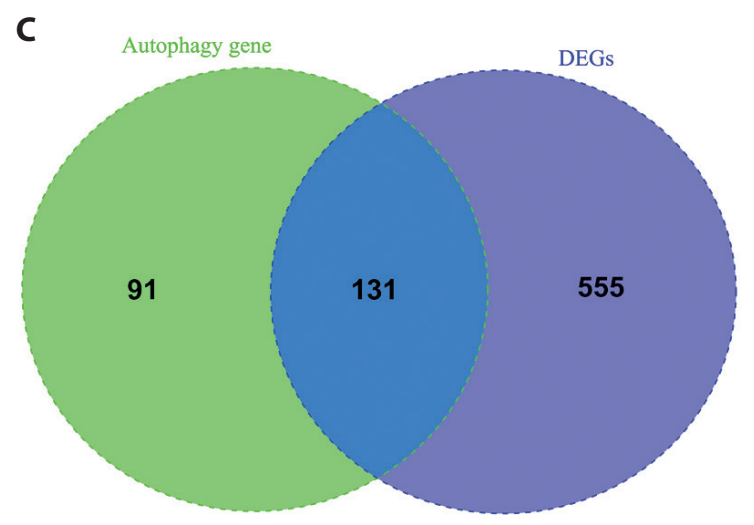

Figure 1. Identification of autophagy-related differentially expressed mRNAs in GBM and LGG samples of CGGA. A. Volcano plot of differentially expressed RNAs. B. Heat map of differentially expressed RNAs. Red, high expression; green, low expression. C. Venn diagram to display the overlap between differentially expressed mRNAs and autophagy-related genes obtained from Human Autophagy Database. FC, fold change; FDR, false discovery rate; GBM, glioblastoma multiforme; LGG, lower-grade glioma; CGGA, the Chinese Glioma Genome Atlas; TCGA, The Cancer Genome Atlas. 
multivariate Cox regression analysis was finally generated to predict the 3-year and 5-year OS. The predictive power of the nomogram was assessed in terms of AUC, concordance index (C-index) and calibration curve.

\section{Results}

\section{Identification of autophagy-related DERs}

HGNC analysis annotated 12,350 protein-encoding mRNAs and 803 lncRNAs shared in CGGA and TCGA databases. Through LIMMA analysis of CGGA dataset, 759 RNAs (including 686 DEGs and 73 DELs) were identified to be differentially expressed between GBM and LGG (Fig. 1A). Hierarchical clustering analysis showed that GBM and LGG samples could be distinctly grouped according to the expressions of the DERs (Fig. 1B). A total of 232 autophagyrelated genes were downloaded from HADb database. Venn diagrams showed 131 of them were intersected with DEGs, which were defined as autophagy-related DEGs for further analysis (Fig. 1C). After calculation of PCC, 54 DELs were considered to be co-expressed with 105 autophagy-related DEGs [such as TMEM72-AS1-ULK2 (unc-51 like autophagy activating kinase 2), WDFY3-AS2-SIRT1 (sirtuin 1)/FoxO3 (forkhead box O3)/TSC1 (TSC complex subunit 1)/HIF1A (hypoxia-inducible factor 1-alpha)] (Fig. 2), suggesting they may be autophagy-related DELs. These 131 DEGs and 54 DELs were considered as autophagy-related DERs and used for further analysis.

\section{Function enrichment analysis for autophagy-related DEGs}

To confirm the autophagy-related functions and other possible roles of our identified autophagy-related genes, function analysis was performed for the autophagy-related DEGs in the co-expression network. As expected, in $23 \mathrm{GO}$ biological process terms enriched, 6 were directly involved in autophagy, including GO:0006914 autophagy [WIPI1 (WD repeat domain, phosphoinositide interacting 1), ULK2, MTOR (mechanistic target of rapamycin kinase)], GO:0016236 macroautophagy [WIPI1, MTOR, MLST8 (MTOR associated protein, LST8 homolog)], GO:0000422 mitophagy (WIPI1), GO:0000045 autophagosome assembly (WIPI1), GO:0016239 positive regulation of macroautophagy (ULK1, HIF1A), GO:0010506 regulation of autophagy (ULK1). Furthermore, these genes also regulated the apoptosis [GO:0006915 apoptotic process: NFKB1 (nuclear factor kappa B subunit 1), PPP1R15A (protein phosphatase 1 regulatory subunit 15A); GO:0043066 negative regulation of apoptotic process: MTOR, SIRT1; GO:0042981 regulation of apoptotic process: CTSB (cathepsin B)], cell cycle ar- rest (GO:0007050: MLST8, MTOR, PPP1R15A) and cellular response to hypoxia (GO:0071456: NFKB1, SIRT1, FoxO3, HIF1A) (Fig. 3A; Table 1). Similar to GO terms, hsa04140:Regulation of autophagy (ULK2) and hsa04210:Apoptosis (NFKB1) KEGG pathways were also enriched for co-expression network genes. In addition, several cancer signaling pathways [such as hsa05200:Pathways in cancer (NFKB1, MTOR, HIF1A), hsa04668:TNF signaling pathway (NFKB1), hsa04066:HIF-1 signaling pathway (NFKB1, MTOR, HIF1A), hsa04150:mTOR signaling pathway (ULK2, MLST8, MTOR, TSC1), hsa04068:FoxO signaling pathway (FOXO3, SIRT1), hsa04151:PI3K-Akt signaling pathway (NFKB1, FOXO3, TSC1, BCL2, MTOR, MLST8) and hsa04012:ErbB signaling pathway (MTOR)] and metabolism-related pathway [hsa05231:Choline metabolism in cancer (TSC1, MTOR, HIF1A)] were also obtained (Fig. 3B; Table 1).

\section{Development of autophagy-related DERs-based risk score}

Univariate Cox regression analysis identified 132 autophagyrelated DERs (including 85 of 131 autophagy-related DEGs and 47 of 54 autophagy-related DELs) were significantly associated with OS $(p<0.05)$. Then, they were included as the variables for the multivariate Cox regression. The results showed 19 DERs (including 14 DEGs and 5 DELs) were independent prognostic factors. Logit regression model was used to further extract the feature genes that distinguished GBM and LGG from these 19 DERs. As a result, 10 genes (including 8 DEGs and 2 DELs) were obtained (Table 2). As shown in Figure 4, these 10 genes could obviously distinguish GBM from LGG, with the accuracy of 0.891 using CGGA dataset and 0.790 using TCGA dataset. Supplementary Table S2 summarized the proportion of variance of each principal component.

The risk score was calculated for each patient according to the following formula: $(-2.419 \times$ expression of TMEM72-AS1 $)+(-0.1293 \times$ expression of WDFY3-AS2 $)+$ $(-0.0009808 \times$ expression of CTSB $)+[-0.0002811 \times$ expression of eukaryotic translation elongation factor 2 (EEF2)] $+[-0.1031 \times$ expression of glutamate ionotropic receptor delta type subunit 2 (GRID2) $]+(-0.01185 \times$ expression of MLST8 $)+(0.04575 \times$ expression of MTOR $)+(-0.1039$ $\times$ expression of NFKB1 $)+(-0.01613 \times$ expression of PPP1R15A $+(0.04084 \times$ expression of WIPI1 $)$. The patients were divided to two groups (low-risk group and high-risk group) using the median as the cut-off. In CGGA training dataset, it was obviously observed that patients with higher risk scores were at an increased risk of developing GBM $(166 / 334(49.7 \%) v s .71 / 334$ (21.3\%); Chi-square $=59.02$, $p<0.001)$. Kaplan-Meier curve analysis showed that patients in the high risk group had a significantly poorer prognosis than those in the low risk group [hazard ratio $(\mathrm{HR})=2.582$, 


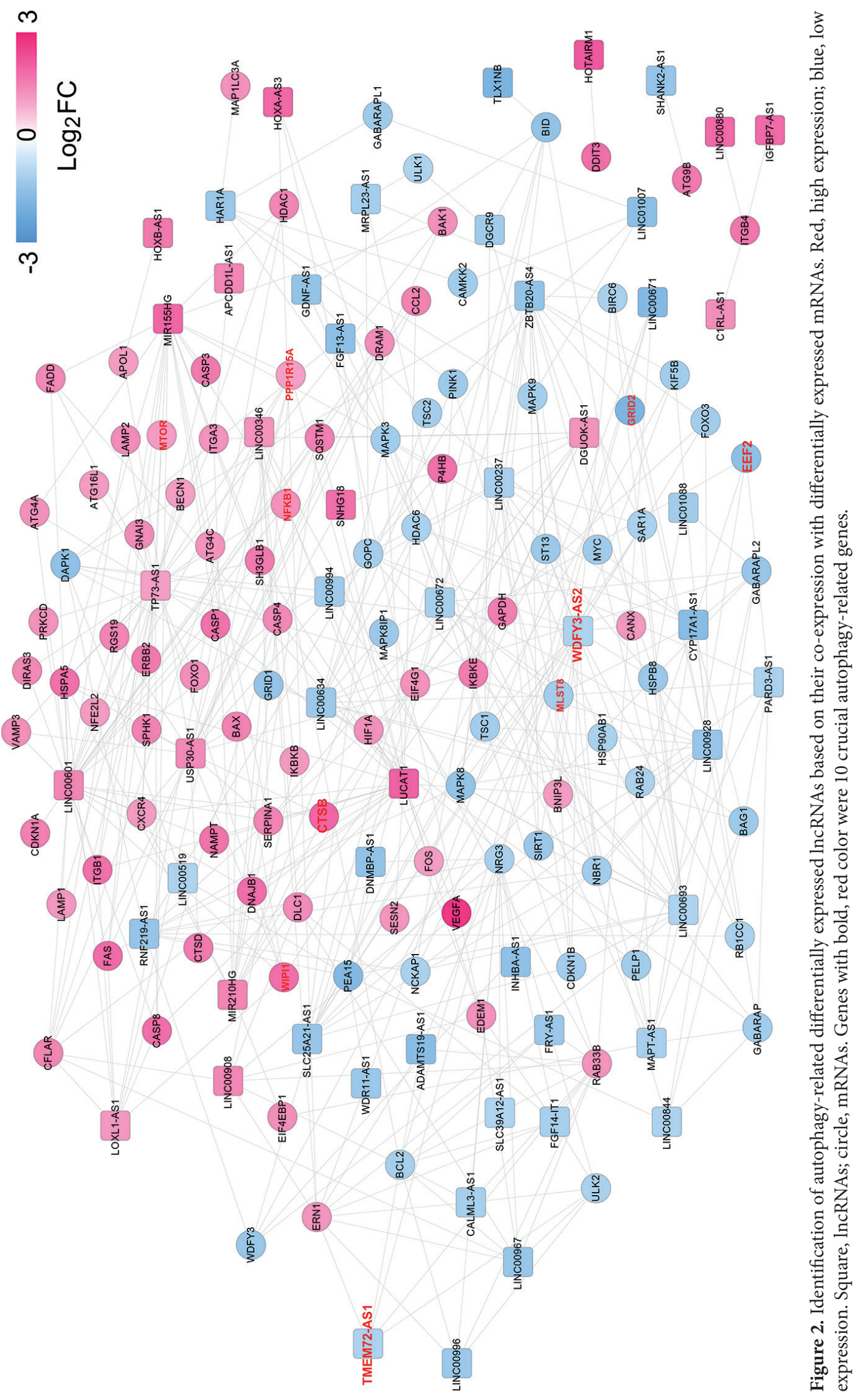


Table 1. Function enrichment results

\begin{tabular}{|c|c|c|c|}
\hline Term & $p$-value & FDR & Genes \\
\hline \multicolumn{4}{|l|}{ Biology Process } \\
\hline GO:0006914 autophagy & $1.53 \mathrm{E}-22$ & $2.54 \mathrm{E}-19$ & $\begin{array}{l}\text { GABARAPL2, GABARAPL1, BECN1, ITGB4, RGS19, FOXO1, } \\
\text { SESN2, WIPI1, GABARAP, RAB33B, LAMP1, ATG4C, SQSTM1, } \\
\text { SH3GLB1, ULK2, ATG4A, RB1CC1, RAB24, CTSD, MTOR, } \\
\text { DRAM1 }\end{array}$ \\
\hline GO:0016236 macroautophagy & $2.42 \mathrm{E}-17$ & $4.01 \mathrm{E}-14$ & $\begin{array}{l}\text { GABARAPL2, GABARAPL1, BECN1, PINK1, WIPI1, GABARAP, } \\
\text { MAP1LC3A, SQSTM1, ULK1, NBR1, RB1CC1, MTOR, MLST8, } \\
\text { ATG16L1, HDAC6 }\end{array}$ \\
\hline GO:0006915 apoptotic process & $5.89 \mathrm{E}-15$ & $9.75 \mathrm{E}-12$ & $\begin{array}{l}\text { DLC1, FOXO1, NFKB1, PEA15, CASP3, CASP4, BAG1, SH3GLB1, } \\
\text { SQSTM1, CXCR4, BCL2, CASP8, FAS, CASP1, CFLAR, BECN1, } \\
\text { BIRC6, FADD, PRKCD, DDIT3, DAPK1, NCKAP1, BAX, MAPK3, } \\
\text { PPP1R15A, DRAM1 }\end{array}$ \\
\hline GO:0000422 mitophagy & $5.82 \mathrm{E}-13$ & $9.64 \mathrm{E}-10$ & $\begin{array}{l}\text { GABARAPL2, ATG9B, GABARAPL1, MAP1LC3A, ATG4C, } \\
\text { SQSTM1, ATG4A, RB1CC1, PINK1, WIPI1 }\end{array}$ \\
\hline GO:0000045 autophagosome assembly & $2.80 \mathrm{E}-12$ & $4.64 \mathrm{E}-09$ & $\begin{array}{l}\text { GABARAPL2, ATG9B, GABARAPL1, MAP1LC3A, ATG4C, } \\
\text { BECN1, ATG4A, RB1CC1, ATG16L1, WIPI1 }\end{array}$ \\
\hline $\begin{array}{c}\text { GO:0043066 negative regulation of } \\
\text { apoptotic process }\end{array}$ & $4.71 \mathrm{E}-12$ & $7.80 \mathrm{E}-09$ & $\begin{array}{l}\text { CFLAR, BECN1, SPHK1, BIRC6, FOXO1, NFKB1, SIRT1, CASP3, } \\
\text { CDKN1A, CDKN1B, BAG1, HDAC1, SQSTM1, BCL2, VEGFA, } \\
\text { BNIP3L, MAPK8, HSPA5, FAS, IKBKB, MYC }\end{array}$ \\
\hline GO:0071456 cellular response to hypoxia & $3.87 \mathrm{E}-10$ & $6.42 \mathrm{E}-07$ & $\begin{array}{l}\text { P4HB, EIF4EBP1, HIF1A, BCL2, VEGFA, BNIP3L, PINK1, FOXO3, } \\
\text { MTOR, NFE2L2, SIRT1 }\end{array}$ \\
\hline $\begin{aligned} \text { GO:0016239 positive regulation of } \\
\text { macroautophagy }\end{aligned}$ & $4.52 \mathrm{E}-09$ & $7.49 \mathrm{E}-06$ & HIF1A, ULK1, SQSTM1, BNIP3L, PINK1, SESN2, SIRT1 \\
\hline $\begin{array}{r}\text { GO:0097192 extrinsic apoptotic signaling } \\
\text { pathway in absence of ligand }\end{array}$ & $5.70 \mathrm{E}-08$ & $9.44 \mathrm{E}-05$ & BAK1, CASP3, BAX, BCL2, FADD, FAS, FOXO3 \\
\hline $\begin{array}{c}\text { GO:0042981 regulation of apoptotic } \\
\text { process }\end{array}$ & $8.40 \mathrm{E}-08$ & $1.39 \mathrm{E}-04$ & $\begin{array}{l}\text { BID, CFLAR, PEA15, CASP4, BAX, CASP8, BNIP3L, FADD, FAS, } \\
\text { CTSB, CASP1, DAPK1 }\end{array}$ \\
\hline $\begin{aligned} \text { GO:0008625 extrinsic apoptotic signaling } \\
\text { pathway via death domain } \\
\text { receptors }\end{aligned}$ & $1.15 \mathrm{E}-07$ & $1.90 \mathrm{E}-04$ & BID, BAX, BCL2, FADD, FAS, GABARAP, DAPK1 \\
\hline GO:0006468 protein phosphorylation & $1.34 \mathrm{E}-07$ & $2.21 \mathrm{E}-04$ & $\begin{array}{l}\text { CCL2, ERBB2, BIRC6, PINK1, PRKCD, CAMKK2, DAPK1, IKBKE, } \\
\text { SQSTM1, ULK1, MAPK3, ERN1, MAPK9, MAPK8, MTOR, IKBKB }\end{array}$ \\
\hline GO:0007050 cell cycle arrest & $2.24 \mathrm{E}-07$ & $3.72 \mathrm{E}-04$ & $\begin{array}{l}\text { CDKN1A, CDKN1B, TSC1, TSC2, ERN1, MLST8, MTOR, PP- } \\
\text { P1R15A, MYC, DDIT3 }\end{array}$ \\
\hline $\begin{array}{r}\text { GO:0071260 cellular response to } \\
\text { mechanical stimulus }\end{array}$ & $2.74 \mathrm{E}-07$ & $4.54 \mathrm{E}-04$ & BAK1, MAPK3, CASP8, NFKB1, MAPK8, FADD, FAS, CASP1 \\
\hline $\begin{array}{c}\text { GO:1900034 regulation of cellular } \\
\text { response to heat }\end{array}$ & $4.01 \mathrm{E}-07$ & $6.63 \mathrm{E}-04$ & $\begin{array}{l}\text { HSP90AB1, BAG1, HSPB8, MAPK3, MLST8, DNAJB1, MTOR, } \\
\text { SIRT1 }\end{array}$ \\
\hline $\begin{array}{c}\text { GO:0006995 cellular response to nitrogen } \\
\text { starvation }\end{array}$ & $9.51 \mathrm{E}-07$ & $1.58 \mathrm{E}-03$ & GABARAPL2, GABARAPL1, MAP1LC3A, BECN1, RB1CC1 \\
\hline $\begin{array}{c}\text { GO:0043065 positive regulation of } \\
\text { apoptotic process }\end{array}$ & $2.52 \mathrm{E}-06$ & $4.17 \mathrm{E}-03$ & $\begin{array}{l}\text { BID, BAK1, SQSTM1, BAX, BNIP3L, FOXO1, MAPK8, FADD, } \\
\text { FAS, FOXO3, SIRT1, ITGB1 }\end{array}$ \\
\hline GO:0097190 apoptotic signaling pathway & $5.04 \mathrm{E}-06$ & $8.35 \mathrm{E}-03$ & BAK1, CASP3, BAX, CASP8, FADD, FAS, DAPK1 \\
\hline $\begin{aligned} \text { GO:0001934 positive regulation of } \\
\text { protein phosphorylation }\end{aligned}$ & $1.39 \mathrm{E}-05$ & $2.30 \mathrm{E}-02$ & $\begin{array}{l}\text { SQSTM1, RB1CC1, ERBB2, MAPK3, VEGFA, PINK1, MTOR, } \\
\text { SIRT1 }\end{array}$ \\
\hline GO:0010506 regulation of autophagy & $1.40 \mathrm{E}-05$ & $2.33 \mathrm{E}-02$ & ULK1, ULK2, CASP1, DRAM1, DAPK1, HDAC6 \\
\hline GO:0009636 response to toxic substance & $1.44 \mathrm{E}-05$ & $2.38 \mathrm{E}-02$ & FOS, CDKN1A, BAX, BCL2, MAPK3, FAS, HDAC6 \\
\hline GO:0050821 protein stabilization & $2.16 \mathrm{E}-05$ & $3.58 \mathrm{E}-02$ & $\begin{array}{l}\text { HSP90AB1, LAMP1, LAMP2, CDKN1A, TSC1, PINK1, PRKCD, } \\
\text { GAPDH }\end{array}$ \\
\hline $\begin{array}{c}\text { GO:0042149 cellular response to glucose } \\
\text { starvation }\end{array}$ & $2.93 \mathrm{E}-05$ & $4.85 \mathrm{E}-02$ & BECN1, SH3GLB1, BCL2, HSPA5, NFE2L2 \\
\hline
\end{tabular}


Table 1. Function enrichment results (continued)

\begin{tabular}{|c|c|c|c|}
\hline Term & $p$-value & FDR & Genes \\
\hline \multicolumn{4}{|l|}{ KEGG Pathway } \\
\hline hsa05200:Pathways in cancer & $22 \mathrm{E}-14$ & $5.09 \mathrm{E}-11$ & $\begin{array}{l}\text { BID, HSP90AB1, GNAI3, ERBB2, FOXO1, NFKB1, ITGB1, FOS, } \\
\text { CASP3, CXCR4, BCL2, CASP8, FAS, MYC, ITGA3, FADD, DAPK1, } \\
\text { CDKN1A, CDKN1B, HIF1A, HDAC1, BAX, VEGFA, MAPK3, } \\
\text { MAPK9, MAPK8, MTOR, IKBKB }\end{array}$ \\
\hline $\begin{array}{l}\text { hsa04141:Protein processing in } \\
\text { endoplasmic reticulum }\end{array}$ & $1.30 \mathrm{E}-10$ & $1.57 \mathrm{E}-07$ & $\begin{array}{l}\text { HSP90AB1, P4HB, CANX, EDEM1, DDIT3, BAK1, BAG1, BAX, } \\
\text { BCL2, ERN1, MAPK9, MAPK8, HSPA5, NFE2L2, DNAJB1, } \\
\text { PPP1R15A, SAR1A }\end{array}$ \\
\hline hsa05161:Hepatitis B & $1.48 \mathrm{E}-10$ & $1.79 \mathrm{E}-07$ & $\begin{array}{l}\text { NFKB1, FADD, IKBKE, FOS, CDKN1A, CASP3, CDKN1B, BAX, } \\
\text { BCL2, CASP8, MAPK3, MAPK9, MAPK8, FAS, IKBKB, MYC }\end{array}$ \\
\hline hsa04140:Regulation of autophagy & $3.22 \mathrm{E}-10$ & $3.89 \mathrm{E}-07$ & $\begin{array}{l}\text { GABARAPL2, GABARAPL1, ATG4C, BECN1, ULK1, ULK2, } \\
\text { ATG4A, ATG16L1, GABARAP }\end{array}$ \\
\hline $\begin{array}{l}\text { hsa05142:Chagas disease (American } \\
\text { trypanosomiasis) }\end{array}$ & $4.10 \mathrm{E}-08$ & $4.95 \mathrm{E}-05$ & $\begin{array}{l}\text { CFLAR, FOS, GNAI3, CCL2, MAPK3, CASP8, MAPK9, NFKB1, } \\
\text { MAPK8, FADD, FAS, IKBKB }\end{array}$ \\
\hline hsa04210:Apoptosis & $4.68 \mathrm{E}-08$ & $5.65 \mathrm{E}-05$ & $\begin{array}{l}\text { BID, CFLAR, CASP3, BAX, BCL2, CASP8, NFKB1, FADD, FAS, } \\
\text { IKBKB }\end{array}$ \\
\hline hsa04668:TNF signaling pathway & $5.53 \mathrm{E}-08$ & $6.67 \mathrm{E}-05$ & $\begin{array}{l}\text { CFLAR, FOS, CASP3, CCL2, MAPK3, CASP8, MAPK9, NFKB1, } \\
\text { MAPK8, FADD, FAS, IKBKB }\end{array}$ \\
\hline hsa05152:Tuberculosis & $1.78 \mathrm{E}-07$ & $2.14 \mathrm{E}-04$ & $\begin{array}{l}\text { BID, SPHK1, FADD, NFKB1, LAMP1, CASP3, LAMP2, BAX, } \\
\text { BCL2, CASP8, MAPK3, CTSD, MAPK9, MAPK8 }\end{array}$ \\
\hline hsa04066:HIF-1 signaling pathway & $2.05 \mathrm{E}-07$ & $2.48 \mathrm{E}-04$ & $\begin{array}{l}\text { EIF4EBP1, CDKN1A, CDKN1B, HIF1A, BCL2, ERBB2, MAPK3, } \\
\text { VEGFA, NFKB1, MTOR, GAPDH }\end{array}$ \\
\hline $\begin{array}{l}\text { hsa04621:NOD-like receptor signaling } \\
\text { pathway }\end{array}$ & $3.11 \mathrm{E}-07$ & $3.76 \mathrm{E}-04$ & $\begin{array}{l}\text { HSP90AB1, CCL2, MAPK3, CASP8, MAPK9, NFKB1, MAPK8, } \\
\text { IKBKB, CASP1 }\end{array}$ \\
\hline hsa04150:mTOR signaling pathway & $4.12 \mathrm{E}-07$ & $4.97 \mathrm{E}-04$ & $\begin{array}{l}\text { EIF4EBP1, TSC1, ULK1, ULK2, TSC2, MAPK3, MLST8, MTOR, } \\
\text { IKBKB }\end{array}$ \\
\hline hsa04068:FoxO signaling & $5.62 \mathrm{E}-07$ & $6.79 \mathrm{E}-04$ & $\begin{array}{l}\text { GABARAPL2, GABARAPL1, CDKN1A, CDKN1B, MAPK3, } \\
\text { FOXO1, MAPK9, MAPK8, FOXO3, IKBKB, SIRT1, GABARAP }\end{array}$ \\
\hline hsa04151:PI3K-Akt signaling pathway & $6.37 \mathrm{E}-07$ & $7.69 \mathrm{E}-04$ & $\begin{array}{l}\text { HSP90AB1, ITGB4, NFKB1, ITGA3, FOXO3, ITGB1, EIF4EBP1, } \\
\text { CDKN1A, CDKN1B, TSC1, BCL2, VEGFA, MAPK3, TSC2, MTOR, } \\
\text { MLST8, IKBKB, MYC }\end{array}$ \\
\hline hsa04012:ErbB signaling & $9.24 \mathrm{E}-07$ & $1.12 \mathrm{E}-03$ & $\begin{array}{l}\text { EIF4EBP1, CDKN1A, CDKN1B, NRG3, ERBB2, MAPK3, MAPK9, } \\
\text { MAPK8, MTOR, MYC }\end{array}$ \\
\hline hsa05215:Prostate cancer & $1.02 \mathrm{E}-06$ & $1.23 \mathrm{E}-03$ & $\begin{array}{l}\text { HSP90AB1, CDKN1A, CDKN1B, BCL2, ERBB2, MAPK3, FOXO1, } \\
\text { NFKB1, MTOR, IKBKB }\end{array}$ \\
\hline hsa05133:Pertussis & $3.06 \mathrm{E}-06$ & $3.70 \mathrm{E}-03$ & $\begin{array}{l}\text { FOS, CASP3, GNAI3, MAPK3, MAPK9, NFKB1, MAPK8, CASP1, } \\
\text { ITGB1 }\end{array}$ \\
\hline hsa05145:Toxoplasmosis & $6.66 \mathrm{E}-06$ & $8.04 \mathrm{E}-03$ & $\begin{array}{l}\text { CASP3, GNAI3, BCL2, MAPK3, CASP8, MAPK9, NFKB1, MAPK8, } \\
\text { IKBKB, ITGB1 }\end{array}$ \\
\hline hsa05210:Colo & & $1.09 \mathrm{E}-02$ & FOS, CASP3, BAX, BCL2, MAPK3, MAPK9, MAPK8, MYC \\
\hline $\begin{array}{l}\text { hsa04932: Non-alcoholic fatty liver disease } \\
\text { (NAFLD) }\end{array}$ & $1.32 \mathrm{E}-05$ & $1.60 \mathrm{E}-02$ & $\begin{array}{l}\text { BID, CASP3, BAX, CASP8, ERN1, MAPK9, NFKB1, MAPK8, FAS, } \\
\text { IKBKB, DDIT3 }\end{array}$ \\
\hline hsa04071:Sphingolipid signaling pathway & $1.36 \mathrm{E}-05$ & $1.64 \mathrm{E}-02$ & $\begin{array}{l}\text { BID, GNAI3, BAX, BCL2, MAPK3, SPHK1, MAPK9, CTSD, } \\
\text { NFKB1, MAPK8 }\end{array}$ \\
\hline hsa04115:p53 signaling pathway & $1.51 \mathrm{E}-05$ & $1.83 \mathrm{E}-02$ & BID, CDKN1A, CASP3, BAX, TSC2, CASP8, FAS, SESN2 \\
\hline hsa05231:Choline metabolism in cancer & $2.84 \mathrm{E}-05$ & $3.43 \mathrm{E}-02$ & $\begin{array}{l}\text { FOS, EIF4EBP1, HIF1A, TSC1, TSC2, MAPK3, MAPK9, MAPK8, } \\
\text { MTOR }\end{array}$ \\
\hline $\begin{array}{l}\text { hsa04620:Toll-like receptor signaling } \\
\text { pathway }\end{array}$ & $4.03 \mathrm{E}-05$ & $4.87 \mathrm{E}-02$ & $\begin{array}{l}\text { IKBKE, FOS, MAPK3, CASP8, MAPK9, NFKB1, MAPK8, FADD, } \\
\text { IKBKB }\end{array}$ \\
\hline
\end{tabular}

GO, Gene Ontology; KEGG, Kyoto Encyclopedia of Genes and Genomes. 
$95 \%$ confidence interval $(\mathrm{CI})=2.092-3.187, p=2.00 \mathrm{e}-16$ ] (Fig. 5A). ROC curve analysis further indicated this risk score had an excellent predictive ability for poor prognosis, with the AUC of 0.840 (Fig. 5C). TCGA dataset was used to further validate the predictive power of risk score identified in CGGA dataset. In line with the results derived from the CGGA dataset, patients with high risk scores were also seen to possess a shorter OS than those with low risk scores (HR $=1.630,95 \% \mathrm{CI}=1.266-2.098, p=1.344 \mathrm{e}-04$ ) (Fig. 5B). The AUC was 0.744 (Fig. 5D).

Univariate and multivariate Cox regression analyses were then performed to evaluate the prognostic independence of the autophagy signature and various clinicopathologic parameters. Consequentially, age (Fig. 6A), recurrence status

A

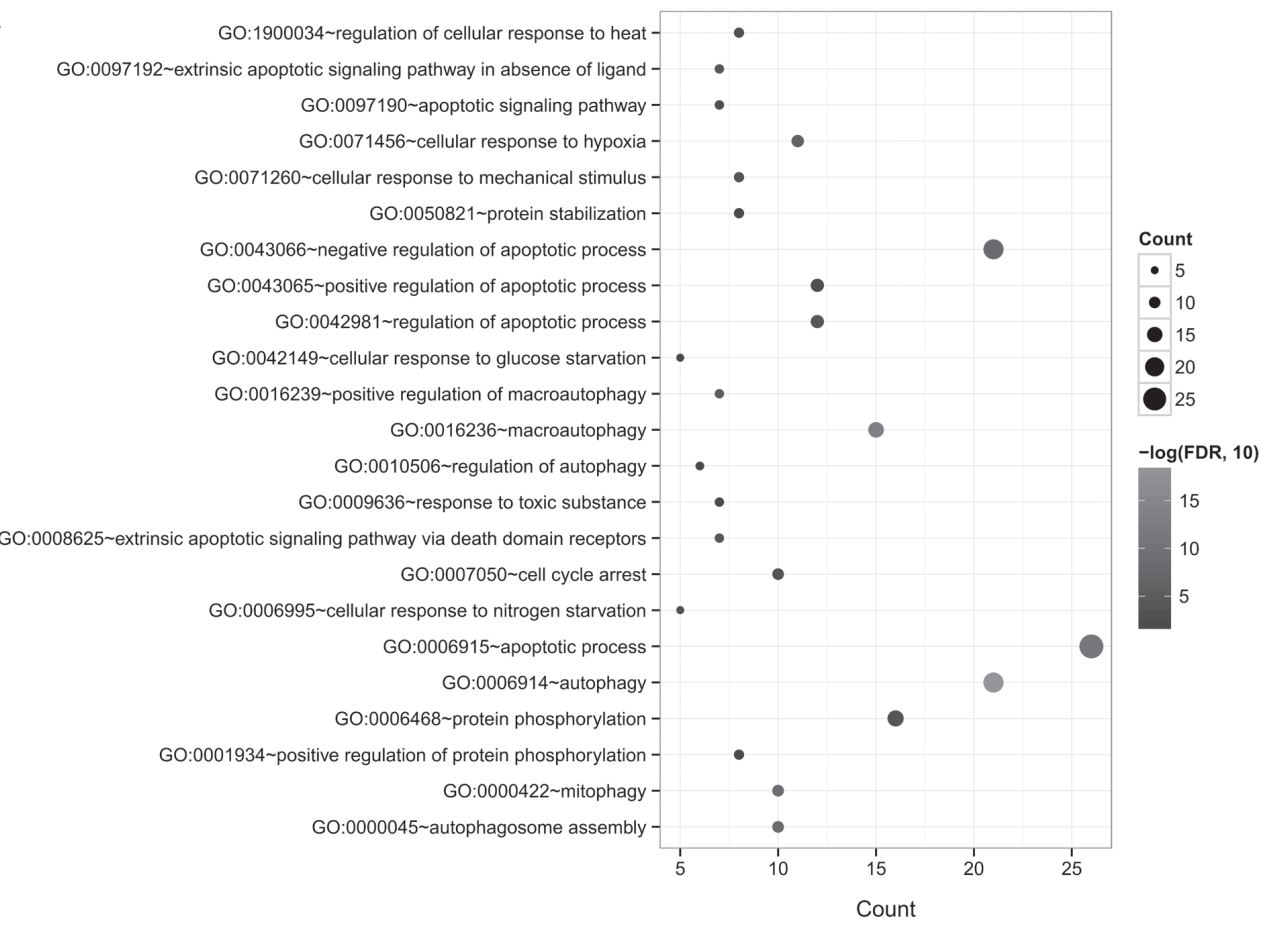

B

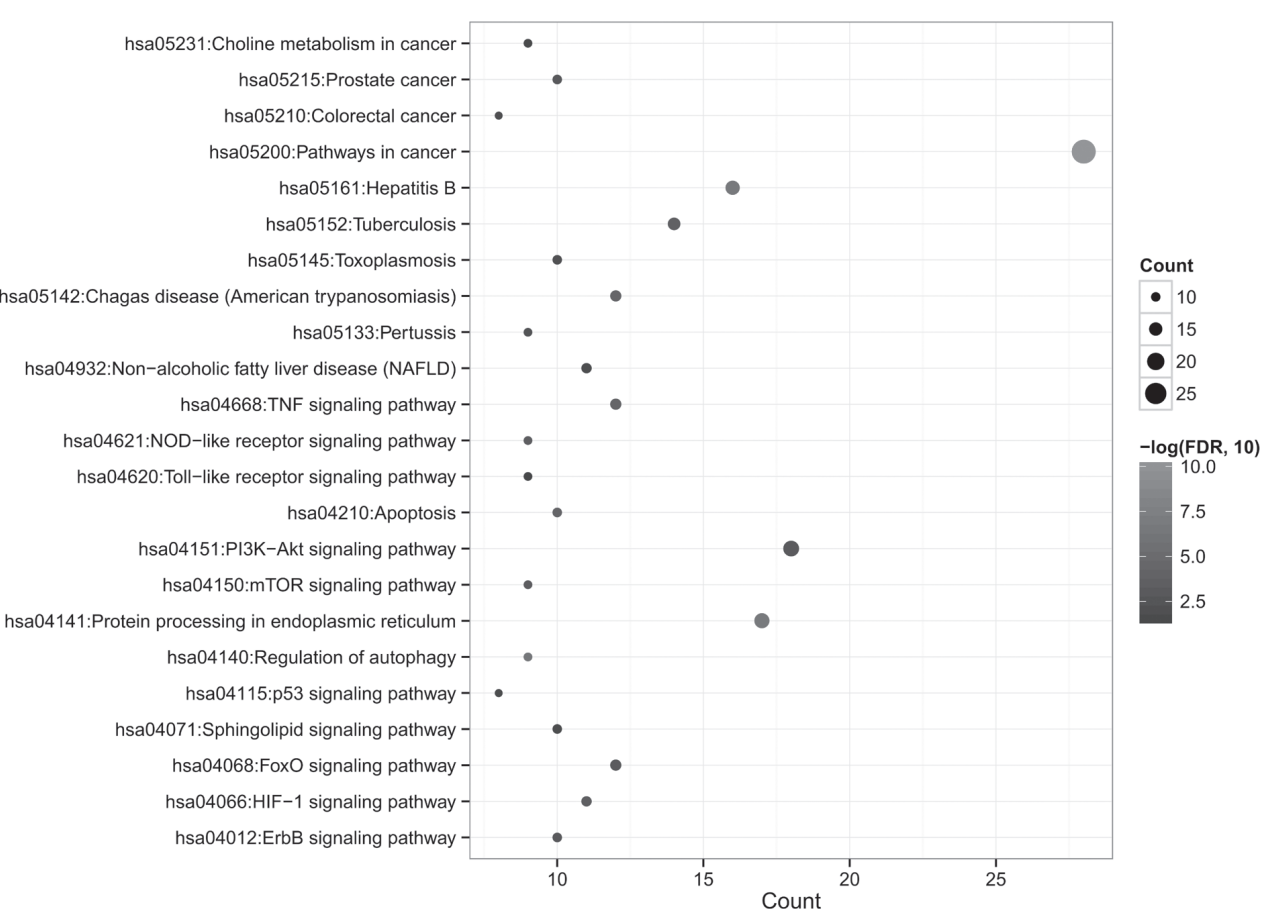

Figure 3. Function enrichment analysis for genes in the co-expression network by DAVID. A. Gene ontology (GO) biological process terms. B. Kyoto Encyclopedia of Genes and Genomes (KEGG). 
(Fig. 6D), radio status, chemo status, IDH mutation status (Fig. 6G) and risk score status were found to be associated with OS in univariate analysis, while only age, recurrence status, IDH mutation status and risk score status were identified as independent prognostic factors in multivariate analysis (Table 3). Furthermore, stratification analysis revealed that the risk score also could divide patients with the same age ( $\geq 45$ years, $p=1.115 \mathrm{e}-09$, Fig. $6 \mathrm{~B} ;<45$ years, $p=5.202 \mathrm{e}-09$, Fig. $6 \mathrm{C}$ ), recurrence status (without recurrence, $\mathrm{p}=1.11 \mathrm{e}-16$, Fig. 6E; recurrence, $p=2.532 \mathrm{e}-04$, Fig. 6F) and IDH status (without mutation, $p=9.323 \mathrm{e}-03$, Fig. $6 \mathrm{H}$; with mutation, $p=3.675 \mathrm{e}-07$, Fig. $6 \mathrm{I}$ ) into the high-risk (shorter OS) and low-risk (longer OS), suggesting the prognostic performance of the risk score was better than those clinical factors. This conclusion was also validated by time-dependent ROC curve (risk score: $\mathrm{AUC}=0.84$ vs. 0.608 , age; 0.599 , recurrence status; 0.672 , IDH mutation status) and C-index (risk score: $0.738 v$ vs. 0.592 , age; 0.596 , recurrence status; 0.661 , IDH mutation status) (Fig. 7A; Table 4). Thus, the risk score was suggested to be incorporated into the clinical factors for prognosis prediction in clinic, based on which a nomogram was built (Fig. 7B). The calibration curves showed that the predicted possibility of OS was similar to the actual OS (Fig. 7C). The AUC (0.879) and C-index (0.773) of nomogram was also higher than age, recurrence status, IDH status and risk score (Fig. 7A).

\section{Discussion}

In the present study, we, for the first time, identified autophagy-related DERs between GBM and LGG and used them to construct the diagnostic and prognostic signature for glioma patients. As a result, 10 signature genes (TMEM72AS1, WDFY3-AS2, CTSB, EEF2, GRID2, MLST8, MTOR, NFKB1, PPP1R15A and WIPI1) were obtained. This signature could obviously distinguish GBM from LGG, with the accuracy of 0.891 using CGGA dataset and 0.790 using TCGA dataset. Its related risk score effectively screened the patients at an increased risk of developing GBM $(49.7 \%$ vs. $21.3 \%, p<0.001$ ) or ones (GBM and possibly progress to GBM) with poor OS. The prognostic accuracy was 0.840 and 0.744 using CGGA and TCGA dataset, respectively. These results were comparable to other risk classification systems established by the lipid metabolism (Wu et al. 2019), pseudogenes (Wang et al. 2019a), necrosis (Wang and Ma 2019) or module (Wang et al. 2019b) related genes that were differentially expressed between GBM and LGG. Furthermore, previous studies indicated mRNA signature outperformed the lncRNA-based signature (Gong et al. 2020), while combined with lncRNAs (Liu Q 2020; Wang 2020a) added the power in predicting prognosis. Thus, we screened the combined IncRNA-mRNA signature and

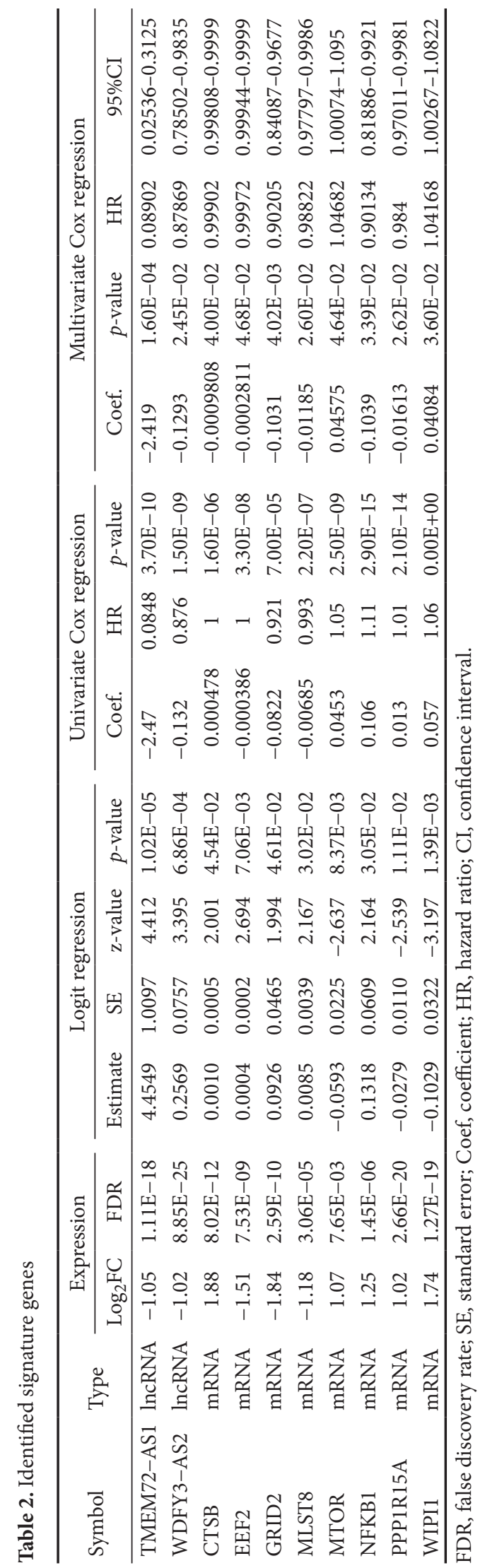


compared the predictive performance of three classifiers. As expected, the AUC (0.84 vs. 0.809; 0.685) and C-index (0.738 vs. $0.696 ; 0.639)$ of IncRNA-mRNA signature were the highest compared with mRNA and lncRNA alone. In line with other signatures reported in glioma patients, the risk score generated in our study was also independent of other clinicopathologic factors (Wang et al. 2019b; Wang et al. 2019c; Wang et al. 2020a) and even superior to the variables that were also independent in multivariate analysis [(risk score: $\mathrm{AUC}=0.84$ vs. 0.608, age; 0.599, recurrence status; 0.672, IDH mutation status) and C-index (risk score: 0.738 vs. 0.592, age; 0.596, recurrence status; 0.661, IDH mutation status)] (Wang et al. 2021). These findings suggested our new risk score may be a promising biomarker for GBM diagnosis and prognosis. In order to obtain better predictive effects in clinic, recent studies recommended to creating a nomogram that integrated the molecular signature with clinical indicators (Wang et al. 2019a, 2019b; Wang et al. 2019c; Wang et al. 2020c). Similarly, a nomogram based on the risk signature, age, recurrence status and IDH mutation status was established in the training cohort. The AUC and C-index reached 0.879 and 0.773 , respectively. Calibration curves showed that there were good agreements between the predicted and observed 3- and 5-year OS. Accordingly, this nomogram may be a clinically simple-to-use tool for prognostic prediction in glioma patients.
A

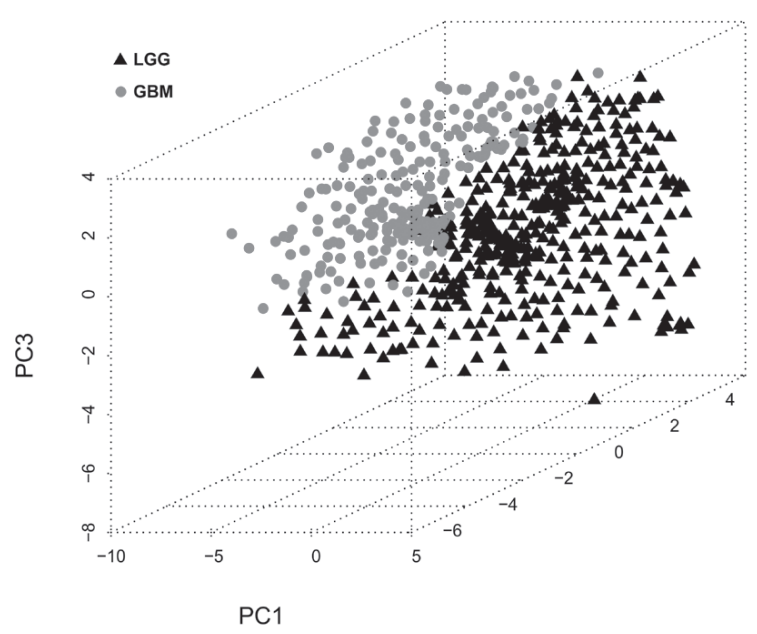

C

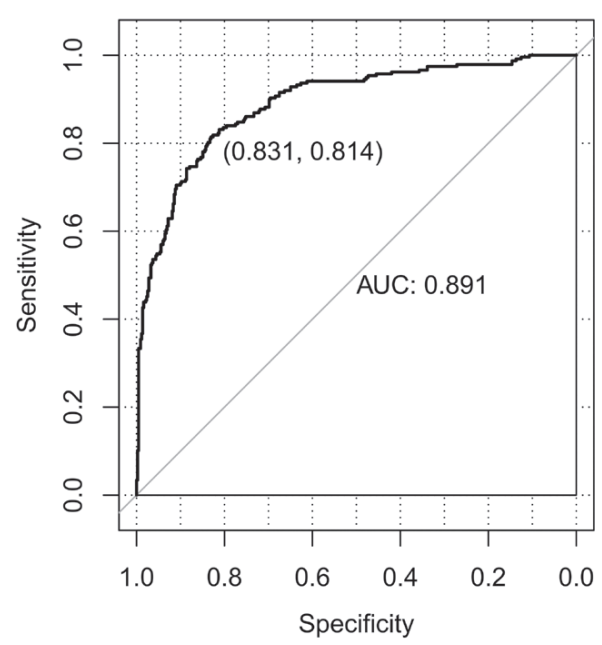

B

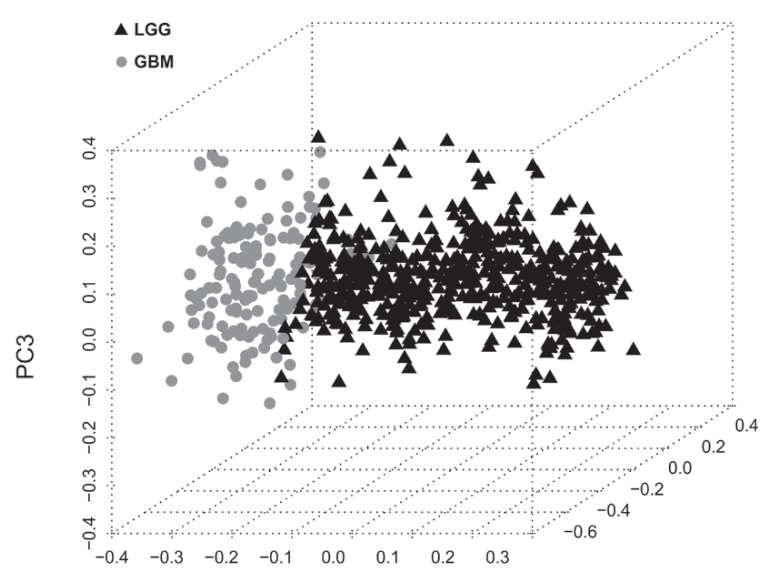

PC1

D

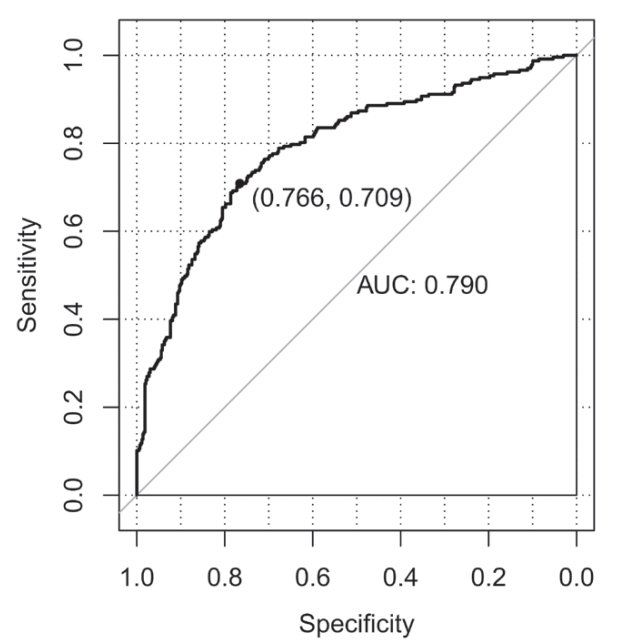

Figure 4. Logit regression model to identify the feature genes to distinguish GBM from LGG. Scatter plot for CGGA (A) and TCGA (B) dataset. Red, GBM samples; black, LGG samples; PC1, PC2, PC3, the first, second, third principal component. Receiver operator characteristic (ROC) curve for CGGA (C) and TCGA (D) dataset. AUC, area under the ROC curve. For more abbreviations, see Fig. 1. 
Table 3. Univariate and multivariate analyses of overall survival

\begin{tabular}{|c|c|c|c|c|c|c|c|}
\hline \multirow{2}{*}{ Variables } & \multirow{2}{*}{$\begin{array}{c}\text { CGGA } \\
(n=668)\end{array}$} & \multicolumn{3}{|c|}{ Univariate analysis } & \multicolumn{3}{|c|}{ Multivariate analysis } \\
\hline & & HR & $95 \% \mathrm{CI}$ & $p$-value & HR & $95 \% \mathrm{CI}$ & $p$-value \\
\hline Age (years, mean $\pm S D$ ) & $43.36 \pm 12.36$ & 1.027 & $1.018-1.035$ & $1.55 \mathrm{E}-09$ & 1.009 & $1.001-1.018$ & $4.83 \mathrm{E}-02$ \\
\hline Gender (Male/Female) & $380 / 288$ & 1.012 & $0.826-1.241$ & $9.07 \mathrm{E}-01$ & - & - & - \\
\hline Recurrence (Yes/No/-) & $259 / 409$ & 2.089 & $1.702-2.564$ & $1.74 \mathrm{E}-12$ & 2.266 & $1.813-2.833$ & $6.74 \mathrm{E}-13$ \\
\hline Radio status (Yes/No/-) & $505 / 110 / 53$ & 1.431 & $1.058-1.935$ & $1.53 \mathrm{E}-02$ & 1.019 & $0.725-1.430$ & $9.16 \mathrm{E}-01$ \\
\hline Chemo status (Yes/No/-) & $454 / 149 / 65$ & 1.506 & $1.154-1.966$ & $1.70 \mathrm{E}-03$ & 0.741 & $0.542-1.015$ & $6.17 \mathrm{E}-02$ \\
\hline $\begin{array}{l}\text { IDH mutation status } \\
\text { (Wildtype/Mutate/-) }\end{array}$ & $277 / 343 / 48$ & 0.309 & $0.249-0.382$ & $2.00 \mathrm{E}-16$ & 0.526 & $0.407-0.679$ & $9.21 \mathrm{E}-07$ \\
\hline Risk score status (High/Low) & $380 / 288$ & 2.582 & $2.092-3.187$ & $2.00 \mathrm{E}-16$ & 1.707 & $1.347-2.165$ & $1.01 \mathrm{E}-05$ \\
\hline
\end{tabular}

HR, hazard ratio; CI, confidence interval; SD, standard deviation; IDH, isocitrate dehydrogenase; CGGA, The Chinese Glioma Genome Atlas.

All these autophagy-related signature mRNAs (CTSB, EEF2, GRID2, MLST8, MTOR, NFKB1, PPP1R15A and WIPI1) had been demonstrated to be associated with the progression of glioma or other cancers. CTSB, which encodes lysosomal cysteine proteinase protein, was reported to be upregulated in GBM cancer cells and stem cell niches (Pucer et al. 2010; Jennewein et al. 2016; Breznik et al. 2018). Overexpression of CTSB decreased chemotherapeutic temozolomide drug-induced glioma cell death and promoted the mesenchymal transition (Ho et al. 2019); while knockdown of CTSB caused cell cycle arrested in G0/G1 phases and enhanced radiosensitivity (Zhang et al. 2018). Elevated CTSB may, like cathepsin L, contributed to radio-resistance in human glioma cells by activation of its downstream NF- $\kappa B$ (Yang et al. 2015). The expression of NF- $\mathrm{KB} 1$ was revealed to be increased with the increasing degree of malignancy in glioma (Yang et al. 2014). PI3K/Akt/ mTOR was a highly activated signaling pathway to induce autophagy for GBM cells (Li et al. 2016). When the PI3K/ Akt/mTOR pathway was blocked by using their corresponding inhibitors, the migration and invasion of GBM U87 cells were shown to be suppressed (Huang et al. 2018). MLST8 combined with mTOR, Rictor, mSin1 and Protor to form the autophagy inhibitor rapamycin-insensitive complex (mTORC2). Increased mTORC2 activity promoted glioma growth and cell motility (Masri et al. 2007). Disruption of the scaffolding function of MLST8 inhibited MTORC2 assembly and its-dependent tumor growth (Hwang et al. 2019). EEF-2 kinase was involved in autophagy by acting as a downstream member of the mTOR signaling pathway (Wu et al. 2009). RNA interference analysis showed silencing of EEF-2 markedly inhibited autophagy and decreased the viability, migration and invasion ability of GBM cells (Wu et al. 2009; Zhang et al. 2011; Liu et al. 2013). PPP1R15A (also known as GADD34) was found to be expressed following DNA damage, a major inducer in hypoxia to activate autophagy (Ito et al. 2015). Hypoxia was reported to upregulate PPP1R15A in glioma cells compared with control (Minchenko et al. 2016). Inhibition of PPP1R15A greatly suppressed anaplastic thyroid carcinoma cell growth (Cao et al. 2019) and potentiated tumor necrosis factor-related apoptosis-inducing ligand (TRAIL)-induced apoptosis of hepatocellular carcinoma cells (Song et al. 2019). Elevated expression of PPP1R15A was associated with poor clinical prognosis (Cao et al. 2019). WIPI1 was suggested to serve as an autophagy biomarker protein (Tsuyuki et al. 2014). It

Table 4. The performance of the nomogram assessed by different classifiers

\begin{tabular}{lccccc}
\hline Model & AUC & C-index & $p$-value & Specificity & Sensitivity \\
\hline Age model & 0.608 & 0.592 & $3.513 \mathrm{E}-09$ & 0.624 & 0.584 \\
Recurrence model & 0.599 & 0.596 & $8.549 \mathrm{E}-14$ & 0.662 & 0.537 \\
IDH mutation model & 0.672 & 0.661 & 0 & 0.755 & 0.588 \\
Clinical model & 0.671 & 0.731 & 0 & 0.685 & 0.66 \\
LncRNAs alone & 0.685 & 0.639 & 0 & 0.525 & 0.793 \\
mRNAs alone & 0.809 & 0.696 & 0 & 0.65 & 0.834 \\
multi-RNAs based model & 0.84 & 0.738 & 0 & 0.743 & 0.795 \\
multi-RNAs combined clinical model & 0.879 & 0.773 & 0 & 0.918 & 0.685 \\
\hline
\end{tabular}

AUC, area under the curve of receiver operating characteristic curve; C-index, concordance index; IDH, isocitrate dehydrogenase. 
A

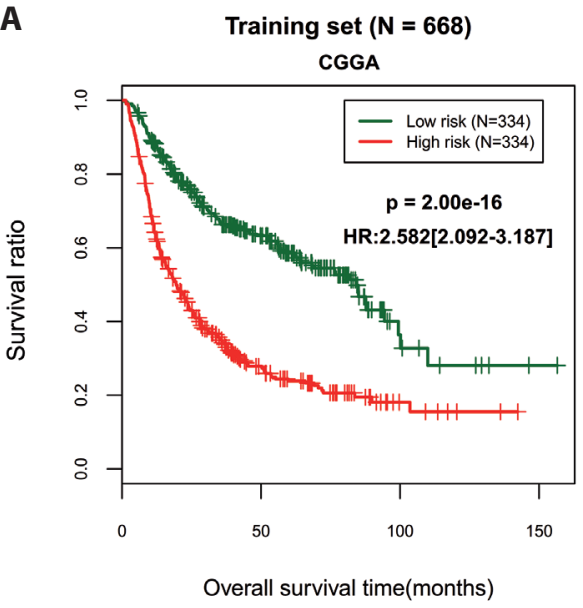

C

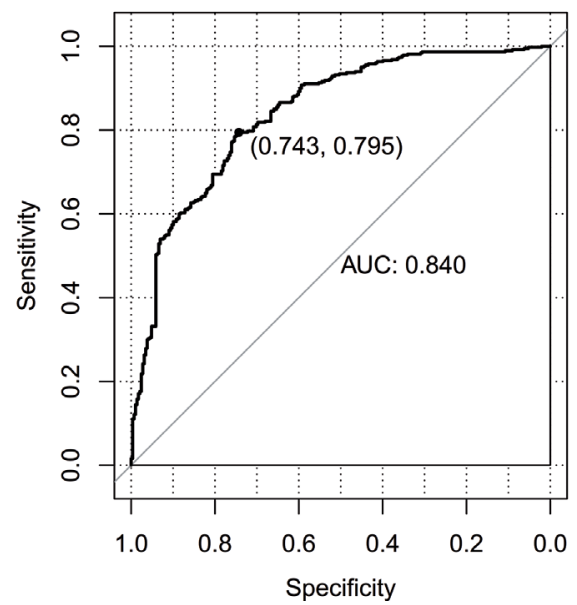

B
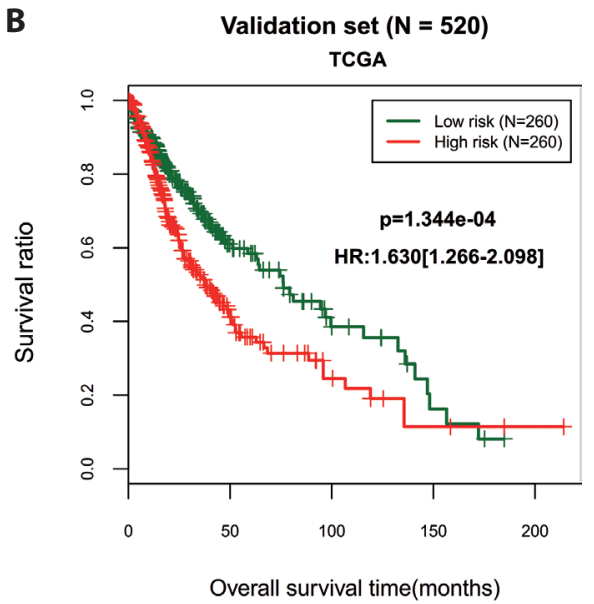

D

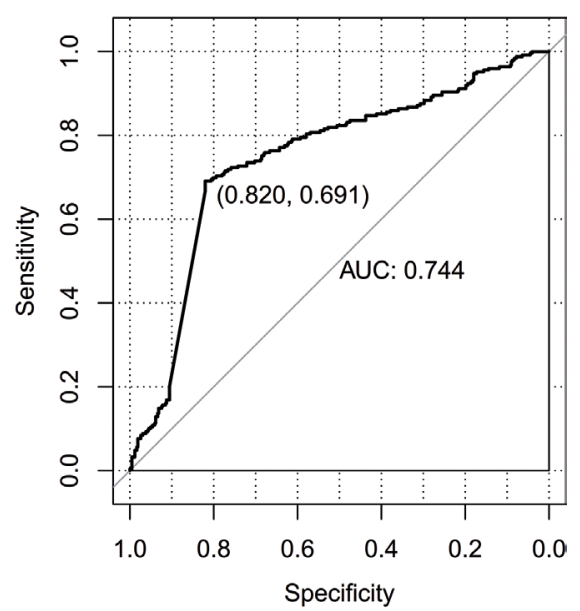

Figure 5. The prognostic performance of the autophagy-related risk score model for glioma patients. Kaplan-Meier survival curve analysis to show the overall survival difference of patients with high risk score and low-risk score in the CGGA (A) and TCGA (B) dataset. Receiver operator characteristic (ROC) to demonstrate the prognostic power of risk score for the overall survival of patients in the CGGA (C) and TCGA (D) dataset. $\mathrm{HR}$, hazard ratio; AUC, area under the ROC curve. For more abbreviations, see Fig. 1. was upregulated in clinical hepatocellular carcinoma (Shi et al. 2016) and melanoma samples (D’Arcangelo and Giampietri 2018). Wang et al. (2021) identified patients with high expression level of GRID2 may have a better prognosis than those with low expression levels. In line with these findings, we also found MTOR, NFKB1, PPP1R15A, WIPI1 and CTSB were higher expressed, while GRID2 was lower expressed in GBM compared with LGG. These genes were enriched in autophagy, apoptosis and cell cycle biological processes or pathways. The expressions of EEF2 and MLST8 were not upregulated as expected, which may be the possible difference between wet and dry experiments or their dual functions.

There were studies to explore lncRNAs that play important roles in glioma by influencing autophagy and its related genes. Luan et al. (2019) used the CGAA dataset to identify 10 autophagy-associated lncRNAs with prognostic value (PCBP1-AS1, TP53TG1, DHRS4-AS1, ZNF674-AS1, GABPB1-AS1, DDX11-AS1, SBF2-AS1, MIR4453HG, MAPKAPK5-AS1 and COX10-AS1). Their established risk score could distinguish the OS between the low-risk group and high-risk group, which was also validated using the TCGA dataset. LncRNA MALAT1 activated autophagy and promoted cell proliferation of glioma cells by upregulating autophagy genes STMN1, RAB5A and ATG4D (Fu et al. 2017), while knockdown of MALAT1 inhibited cell migration and invasion of glioma cells by suppressing autophagy through regulation of autophagy gene GOLM1 (Ma et al. 2020). Elevated lncRNA AC023115.3 and growth arrest-specific 5 (GAS5) in human GBM cells were demonstrated to promote cisplatin-induced apoptosis by inhibiting autophagy (Ma et al. 2017; Huo and Chen 2019). However, autophagy-related lncRNAs remain rarely reported. In our study, we identified TMEM72-AS1 and WDFY3-AS2 may be important biomarkers for glioma patients to separate GBM from LGG and predict their prognosis. WDFY3-AS2 was previously reported in the study of Wu et al. (2018) who found WDFY3-AS2 was one downregulated lncRNA in GBM compared with LGG and patients with high WDFY3-AS2 expression had longer OS than the low expression ones. These conclusions were also demonstrated in our study. However, compared with 
individual WDFY3-AS2 (Wu et al. 2018), our combined signature (including WDFY3-AS2 and other genes) may be more effective for clinical application of prognosis prediction (AUC $=0.840$ vs. 0.796). More importantly, the study of Wu et al. (2018) did not provide the spe- cific downstream target genes to explain the functions of WDFY3-AS2. Our study, for the first time, speculated WDFY3-AS2 may function by co-expressing with SIRT1, FoxO3a, TSC1 and HIF1A. These co-expressed genes have been demonstrated to act as suppressor genes for the
A

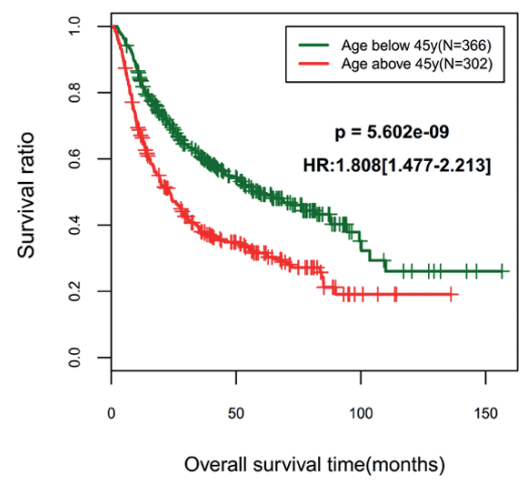

D

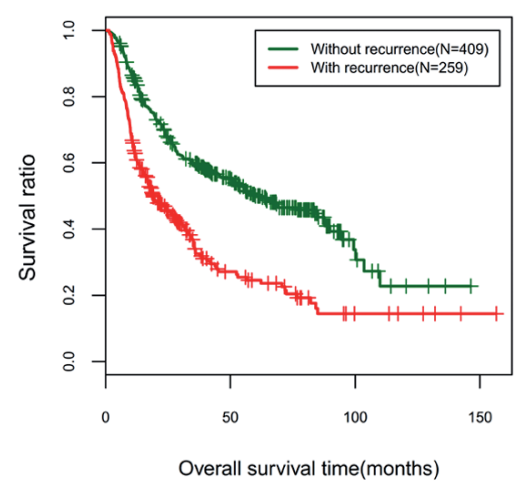

$\mathbf{G}$

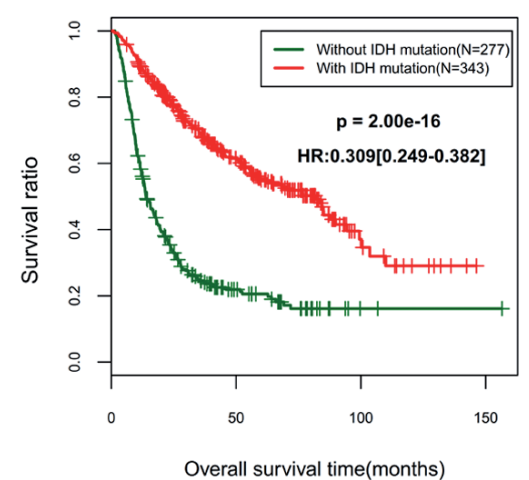

B

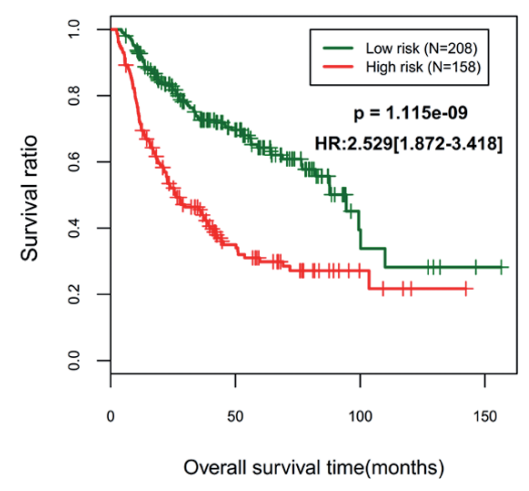

E

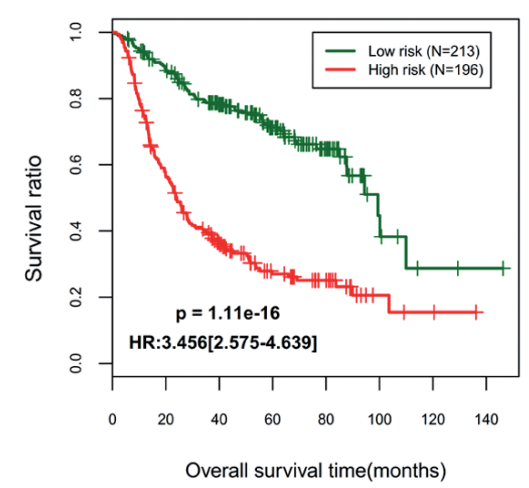

H

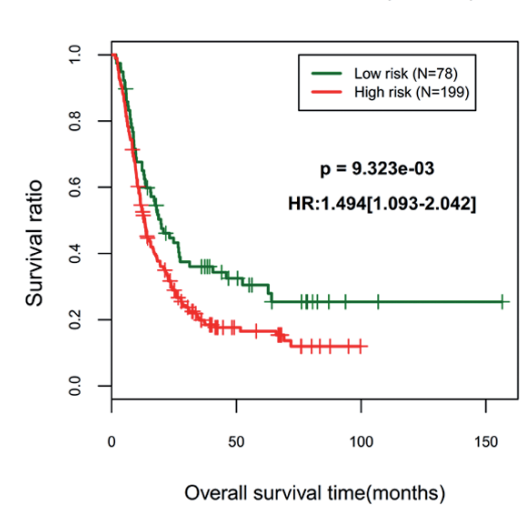

C

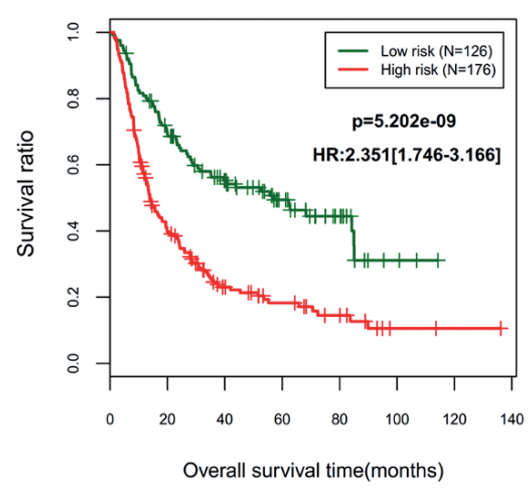

$\mathbf{F}$

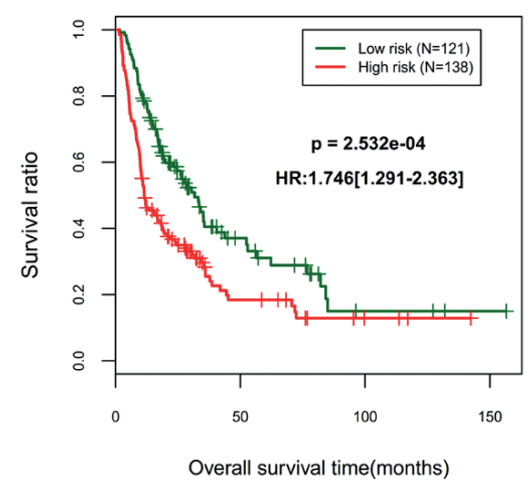

I

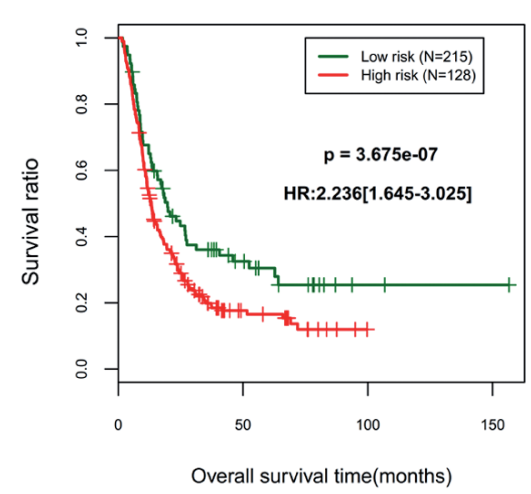

Figure 6. Risk stratification model based on age, recurrence and IDH mutation for glioma patients. The association of age (A), recurrence (D) and IDH mutation (G) with overall survival. The prognosis of patients in the age range (below 45 years (B) and above 45 years (C)) according to the risk score. The prognosis of patients in the different recurrence status according to the risk score (E and $\mathbf{F})$. The prognosis of patients without (H) and with (I) IDH mutation status according to the risk score. HR, hazard ratio; y, year; IDH, isocitrate dehydrogenase. 

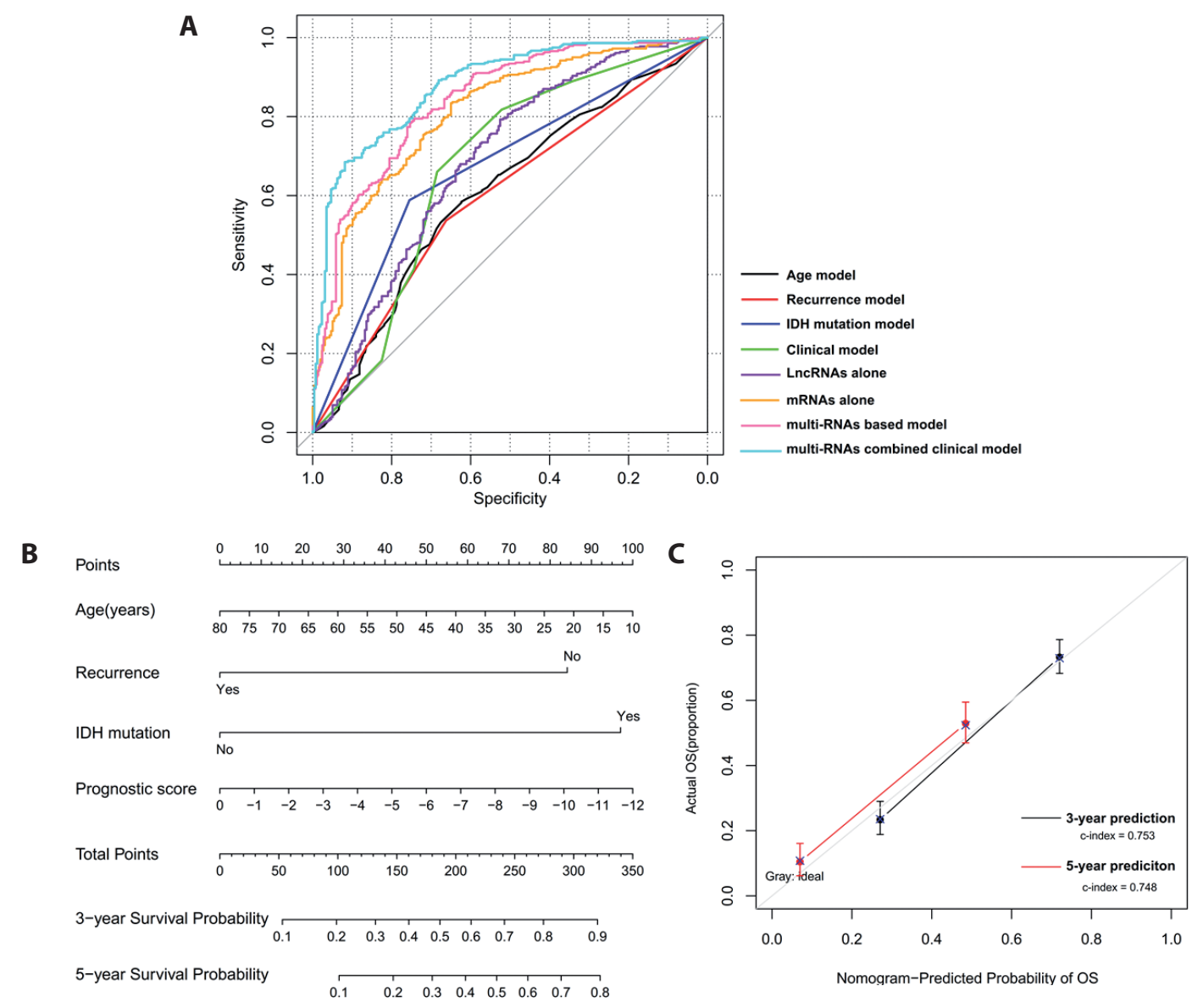

Figure 7. A prognostic nomogram to predict the survival probability of patients with glioma. A. Receiver operator characteristic curve to demonstrate the superiority of risk score for prognosis prediction to other clinical factors. B. A prognostic nomogram. C. Calibration curves.

progression of GBM or other cancers. It was reported that SIRT1 hindered autophagy and GBM growth by mediating the deacetylation of p21-activated kinase 1 (PAK1) at K420 and suppressing the PAK1-ATG5 (autophagy related 5) pathway (Feng et al. 2021). FoxO3a was found to be a negative regulator of autophagy in multiple cancer cells (Zhu et al. 2014). Increased FOXO3a inhibited autophagy and stimulated transcription of the pro-apoptotic BBC3/PUMA gene to cause apoptosis sensitization, thus reducing tumor burden (Fitzwalter et al. 2018). SIRT1 exerted inhibitory activities on chemoresistance and cancer stemness of gastric cancer by initiating the transcription of FOXO3 (An et al. 2020). TSC1, a negative regulator of mTOR signaling, was downregulated in high-grade serous ovarian carcinoma compared with normal fimbria and low stage patients. Ectopic expression of TSC1 could block cell proliferation, migration and autophagy (Wang et al. 2017). Hypoxia induced autophagy in glioma LN229 cells through upregulating the expression of HIF1A expression. Knockout of HIF1A inhibited cell motility and chemosen- sitivity (Hu et al. 2012; Huang et al. 2019). Furthermore, it was observed that the concentrations of betaine, phosphocholine and choline were lowed in HIF1B-deficient hepatoma compared with wide type (Griffiths et al. 2002); while these choline metabolism products were elevated in glioma (Gillies et al. 1994) and targeted drugs may treat GBM by decreasing phosphocholine and choline kinase a (Venkatesh et al. 2012), suggesting HIF1A may also be involved in the glioma progression by influencing choline metabolism. In line with these studies, SIRT1, FoxO3a and TSC1 were also shown to be downregulated, while HIF1A was upregulated in GBM compared with LGG. These genes were not only autophagy-related, but also enriched in various pathways [hsa04066:HIF-1 signaling pathway (HIF1A), hsa04068:FoxO signaling pathway (FOXO3, SIRT1), hsa04150:mTOR signaling pathway (TSC1) and hsa05231: Choline metabolism in cancer (TSC1, HIF1A)]. The roles of TMEM72-AS1 had not been illustrated for any diseases. Our study, for the first time, predicted it may be one crucial lncRNA for malignant progression of glioma 
by regulating autophagy-related ULK2. The protein expression level of ULK2 was observed to be significantly lower in NSCLC cases (Cheng et al. 2019) and glioma (Shukla et al. 2014) compared with control. Overexpression of ULK2 significantly inhibited the proliferation of A549 and H460 cells and improved the chemosensitivity to cisplatin and etoposide. Overexpression of ULK2 also suppressed tumor volume in vivo (Cheng et al. 2019). Our expression level of ULK2 in GBM was consistent with the study of Cheng et al. (2019) and Shukla et al. (2014). However, unfortunately, several studies suggested ULK2 may also induce autophagy, not inhibit autophagy in cancer (John Shukla et al. 2014; Clotaire et al. 2016; Cheng et al. 2019). This may be, on one hand, attributed to the dual functions of autophagy; on the other hand, may be associated with the response loop (Wang et al. 2018). Thus, the combination treatment of ULK overexpression with autophagy inhibitors may potentially be a more effective therapeutic strategy for GBM.

There were some limitations in this study. First, the proposed signature was established and validated using the public datasets. The newly hospitalized GBM and LGG patients in our hospital should be enrolled to further investigate the performance of the signature. Second, functional experiments should be performed to explore the relationship between our identified lncRNAs and mRNAs (TMEM72AS1-ULK2, WDFY3-AS2-SIRT1/FoxO3/TSC1) and their associations with progression of glioma.

\section{Conclusion}

Our study successfully developed a novel 10-gene signature constituted by $2 \operatorname{lncRNAs}$ and 8 autophagy-related genes. This signature distinguished GBM from LGG and predicted OS in patients with glioma. The established nomogram that integrated the risk score and clinical parameters may be more effective to aid the clinical decision making of personalized treatment.

Acknowledgements. This study was supported by the Science and Technology of Jilin Province (No. 20200201470JC).

Availability of data and materials. All data were collected from CGGA (http://www.cgga.org.cn) and TCGA (https://gdc-portal. nci.nih.gov/) and databases.

Authors' contributions. BW, LW and JWZ conceived and designed the original study. BW and LW analyzed the data and drafted the manuscript. JWZ contributed to the interpretation of data and revised the manuscript. All authors read and approved the final manuscript.

Conflict of interest. The authors declare that they have no competing interests.

\section{References}

Almutrafi A, Bashawry Y, AlShakweer W, Al-Harbi M, Altwairgi A, Al-Dandan S (2020): The epidemiology of primary central nervous system tumors at the National Neurologic Institute in Saudi Arabia: A Ten-year single-institution study. J. Cancer Epidemiol. 2020, 1429615

https://doi.org/10.1155/2020/1429615

An Y, Wang B, Wang X, Dong G, Jia J, Yang Q (2020): SIRT1 inhibits chemoresistance and cancer stemness of gastric cancer by initiating an AMPK/FOXO3 positive feedback loop. Cell Death Dis. 11, 115 https://doi.org/10.1038/s41419-020-2308-4

Araghi M, Roshandel G, Hasanpour-Heidari S, Fazel A, Sedaghat SM, Pourkhani A, Kazeminejhad V, Miranda-Filho A, Bray F, Arnold M (2020): Incidence of malignant brain and central nervous system tumors in Golestan, Iran, 2004-2013. Arch. Iran Med. 23, 1-6

Biterge-Sut B (2020): A comprehensive analysis of the angiogenesisrelated genes in glioblastoma multiforme vs. brain lower grade glioma. Arq. Neuropsiquiatr. 78, 34-38 https://doi.org/10.1590/0004-282x20190131

Breznik B, Limbaeck Stokin C, Kos J, Khurshed M, Hira VVV, Bošnjak R, Lah TT, Van Noorden CJF (2018): Cysteine cathepsins $\mathrm{B}, \mathrm{X}$ and $\mathrm{K}$ expression in peri-arteriolar glioblastoma stem cell niches. J. Mol. Histol. 49, 481-497 https://doi.org/10.1007/s10735-018-9787-y

Cao X, Dang L, Zheng X, Lu Y, Lu Y, Ji R, Zhang T, Ruan X, Zhi J, Hou X, et al. (2019): Targeting super-enhancer-driven oncogenic transcription by CDK7 inhibition in anaplastic thyroid carcinoma. Thyroid 29, 809-823

https://doi.org/10.1089/thy.2018.0550

Cheng H, Yang ZT, Bai YQ, Cai YF, Zhao JP (2019): Overexpression of Ulk2 inhibits proliferation and enhances chemosensitivity to cisplatin in non-small cell lung cancer. Oncol. Lett. 17, 79-86 https://doi.org/10.3892/ol.2018.9604

Cj P, Hv E, Vijayakurup V, R Menon G, Nair S, Gopala S (2019): High LC3/beclin expression correlates with poor survival in glioma: a definitive role for autophagy as evidenced by in vitro autophagic flux. Pathol. Oncol. Res. 25, 137-148 https://doi.org/10.1007/s12253-017-0310-7

D'Arcangelo D, Giampietri C (2018): WIPI1, BAG1, and PEX3 autophagy-related genes are relevant melanoma markers. Oxid. Med. Cell Longev. 2018, 1471682 https://doi.org/10.1155/2018/1471682

Dennis G, Sherman BT, Hosack DA, Yang J, Gao W, Lane HC, Lempicki RA (2003): DAVID: Database for annotation, visualization, and integrated discovery. Genome Biol. 4, P3 https://doi.org/10.1186/gb-2003-4-5-p3

Feng X, Zhang H, Meng L, Song H, Zhou Q, Qu C, Zhao P, Li Q, Zou C, Liu X, Zhang Z (2021): Hypoxia-induced acetylation of PAK1 enhances autophagy and promotes brain tumorigenesis via phosphorylating ATG5. Autophagy 17, 723-742 https://doi.org/10.1080/15548627.2020.1731266

Fitzwalter BE, Towers CG, Sullivan KD, Andrysik Z, Hoh M, Ludwig M, O'Prey J, Ryan KM, Espinosa JM, Morgan MJ, Thorburn A (2018): Autophagy inhibition mediates apoptosis 
sensitization in cancer therapy by relieving FOXO3a turnover. Dev. Cell. 44, 555-565.e553 https://doi.org/10.1016/j.devcel.2018.02.014

Fu Z, Luo W, Wang J, Peng T, Sun G, Shi J, Li Z, Zhang B (2017): Malat1 activates autophagy and promotes cell proliferation by sponging miR-101 and upregulating STMN1, RAB5A and ATG4D expression in glioma. Biochem. Biophys. Res. Commun. 492, 480-486 https://doi.org/10.1016/j.bbrc.2017.08.070

Gillies RJ, Barry JA, Ross BD (1994): In vitro and in vivo 13C and 31P NMR analyses of phosphocholine metabolism in rat glioma cells. Magn. Reson. Med. 32, 310-318 https://doi.org/10.1002/mrm.1910320306

Gong Z, Hong F, Wang H, Zhang X, Chen J (2020): An eightmRNA signature outperforms the lncRNA-based signature in predicting prognosis of patients with glioblastoma. BMC Med. Genet. 21, 56 https://doi.org/10.1186/s12881-020-0992-7

Griffiths JR, McSheehy PM, Robinson SP, Troy H, Chung YL, Leek RD, Williams KJ, Stratford IJ, Harris AL, Stubbs M (2002): Metabolic changes detected by in vivo magnetic resonance studies of HEPA-1 wild-type tumors and tumors deficient in hypoxia-inducible factor-1beta (HIF-1beta): evidence of an anabolic role for the HIF-1 pathway. Cancer Res. 62, 688-695

Ho KH, Cheng CH, Chou CM, Chen PH, Liu AJ, Lin CW, Shih CM, Chen KC (2019): miR-140 targeting CTSB signaling suppresses the mesenchymal transition and enhances temozolomide cytotoxicity in glioblastoma multiforme. Pharmacol. Res. 147, 104390 https://doi.org/10.1016/j.phrs.2019.104390

Hu YL, DeLay M, Jahangiri A, Molinaro AM, Rose SD, Carbonell WS, Aghi MK (2012): Hypoxia-induced autophagy promotes tumor cell survival and adaptation to antiangiogenic treatment in glioblastoma. Cancer Res. 72, 1773-1783 https://doi.org/10.1158/0008-5472.CAN-11-3831

Huang W, Ding X, Ye H, Wang J, Shao J, Huang T (2018): Hypoxia enhances the migration and invasion of human glioblastoma U87 cells through PI3K/Akt/mTOR/HIF-1 $\alpha$ pathway. Neuroreport 29, 1578-1585 https://doi.org/10.1097/WNR.0000000000001156

Huang S, Qi P, Zhang T, Li F, He X (2019): The HIF-1 $\alpha /$ miR-224-3p/ ATG5 axis affects cell mobility and chemosensitivity by regulating hypoxia-induced protective autophagy in glioblastoma and astrocytoma. Oncol. Rep. 41, 1759-1768 https://doi.org/10.3892/or.2018.6929

Huo JF, Chen XB (2019): Long noncoding RNA growth arrestspecific 5 facilitates glioma cell sensitivity to cisplatin by suppressing excessive autophagy in an mTOR-dependent manner. J. Cell Biochem. 120, 6127-6136 https://doi.org/10.1002/jcb.27900

Hwang Y, Kim LC, Song W, Edwards DN (2019): Disruption of the scaffolding function of mLST8 selectively inhibits mTORC2 assembly and function and suppresses mTORC2-dependent tumor growth in vivo. Cancer Res. 79, 3178-3184 https://doi.org/10.1158/0008-5472.CAN-18-3658

Ito S, Tanaka Y, Oshino R, Aiba K, Thanasegaran S, Nishio N, Isobe K (2015): GADD34 inhibits activation-induced apoptosis of macrophages through enhancement of autophagy. Sci. Rep. 5,8327 https://doi.org/10.1038/srep08327

Jennewein L, Ronellenfitsch MW, Antonietti P, Ilina EI, Jung J, Stadel D, Flohr LM, Zinke J, von Renesse J, Drott U, et al. (2016): Diagnostic and clinical relevance of the autophago-lysosomal network in human gliomas. Oncotarget 7, 20016-20032 https://doi.org/10.18632/oncotarget.7910

Jiang T, Wu Z (2018): Immunohistochemical assessment of autophagic protein LC3B and p62 levels in glioma patients. Int. J. Clin. Exp. Pathol. 11, 862-868

John Clotaire DZ, Zhang B, Wei N, Gao R, Zhao F, Wang Y, Lei M, Huang W (2016): MiR-26b inhibits autophagy by targeting ULK2 in prostate cancer cells. Biochem. Biophys. Res. Commun. 472, 194-200

https://doi.org/10.1016/j.bbrc.2016.02.093

Li X, Wu C, Chen N, Gu H, Yen A, Cao L, Wang E, Wang L (2016): $\mathrm{PI} 3 \mathrm{~K} / \mathrm{Akt} / \mathrm{mTOR}$ signaling pathway and targeted therapy for glioblastoma. Oncotarget 7, 33440-33450 https://doi.org/10.18632/oncotarget.7961

Li X, He S, Ma B (2020): Autophagy and autophagy-related proteins in cancer. Mol. Cancer 19, 12 https://doi.org/10.1186/s12943-020-1138-4

Liu Q, Wang Z, Kong X, Wang X, Qi Y, Gao R, Fang Y, Wang J (2020): A Novel prognostic signature of mRNA-lncRNA in breast cancer. DNA Cell Biol. 39, 671-682 https://doi.org/10.1089/dna.2019.5223

Liu XY, Zhang L, Wu J, Zhou L, Ren YJ, Yang WQ, Ming ZJ, Chen B, Wang J, Zhang Y, Yang JM (2013): Inhibition of elongation factor-2 kinase augments the antitumor activity of Temozolomide against glioma. Plos One 8, e81345 https://doi.org/10.1371/journal.pone.0081345

Luan F, Chen W, Chen M, Yan J, Chen H, Yu H, Liu T, Mo L (2019): An autophagy-related long non-coding RNA signature for glioma. Febs. Open Bio. 9, 653-667 https://doi.org/10.1002/2211-5463.12601

Ma B, Yuan Z, Zhang L, Lv P, Yang T, Gao J, Pan N, Wu Q, Lou J, Han C, Zhang B (2017): Long non-coding RNA AC023115.3 suppresses chemoresistance of glioblastoma by reducing autophagy. Biochim. Biophys. Acta 1864, 1393-1404

https://doi.org/10.1016/j.bbamcr.2017.05.008

Ma R, Zhang BW, Zhang ZB, Deng QJ (2020): LncRNA MALAT1 knockdown inhibits cell migration and invasion by suppressing autophagy through miR-384/GOLM1 axis in glioma. Eur. Rev. Med. Pharmacol. Sci. 24, 2601-2615

Masri J, Bernath A, Martin J, Jo OD, Vartanian R, Funk A, Gera J (2007): mTORC2 activity is elevated in gliomas and promotes growth and cell motility via overexpression of rictor. Cancer Res. 67, 11712-11720 https://doi.org/10.1158/0008-5472.CAN-07-2223

Minchenko OH, Kryvdiuk IV, Riabovol OO, Minchenko DO, Danilovskyi SV, Ratushna OO (2016): Inhibition of IRE1 modifies the hypoxic regulation of GADD family gene expressions in U87 glioma cells. Ukr. Biochem. J. 88, 25-34 https://doi.org/10.15407/ubj88.02.025

Ostrom QT, Cote DJ, Ascha M, Kruchko C, Barnholtz-Sloan JS (2018): Adult glioma incidence and survival by race or ethnicity in the United States from 2000 to 2014. JAMA Oncol. 4, 1254-1262 
https://doi.org/10.1001/jamaoncol.2018.1789

Povey S, Lovering R, Bruford E, Wright M, Lush M, Wain H (2001): The HUGO Gene Nomenclature Committee (HGNC). Hum. Genet. 109, 678-680 https://doi.org/10.1007/s00439-001-0615-0

Pucer A, Castino R, Mirković B, Falnoga I, Slejkovec Z, Isidoro C, Lah TT (2010): Differential role of cathepsins B and L in autophagy-associated cell death induced by arsenic trioxide in U87 human glioblastoma cells. Biol. Chem. 391, 519-531 https://doi.org/10.1515/bc.2010.050

Ritchie ME, Phipson B, Wu D, Hu Y, Law CW, Shi W, Smyth GK (2015): limma powers differential expression analyses for RNAsequencing and microarray studies. Nucleic. Acids. Res. 43, e47 https://doi.org/10.1093/nar/gkv007

Shi L, Zhang W, Zou F, Mei L, Wu G, Teng Y (2016): KLHL21, a novel gene that contributes to the progression of hepatocellular carcinoma. BMC Cancer 16, 815 https://doi.org/10.1186/s12885-016-2851-7

Shukla S, Patric IR, Patil V, Shwetha SD, Hegde AS, Chandramouli BA, Arivazhagan A, Santosh V, Somasundaram K (2014): Methylation silencing of ULK2, an autophagy gene, is essential for astrocyte transformation and tumor growth. J. Biol. Chem. 289, 22306-22318 https://doi.org/10.1074/jbc.M114.567032

Song P, Yang S, Hua H, Zhang H, Kong Q, Wang J, Luo T, Jiang Y (2019): The regulatory protein GADD34 inhibits TRAILinduced apoptosis via TRAF6/ERK-dependent stabilization of myeloid cell leukemia 1 in liver cancer cells. J. Biol. Chem. 294, 5945-5955 https://doi.org/10.1074/jbc.RA118.006029

Tsuyuki S, Takabayashi M, Kawazu M, Kudo K, Watanabe A, Nagata Y, Kusama Y, Yoshida K (2014): Detection of WIPI1 mRNA as an indicator of autophagosome formation. Autophagy 10, 497-513 https://doi.org/10.4161/auto.27419

Venkatesh HS, Chaumeil MM, Ward CS, Haas-Kogan DA, James CD, Ronen SM (2012): Reduced phosphocholine and hyperpolarized lactate provide magnetic resonance biomarkers of PI3K/Akt/mTOR inhibition in glioblastoma. Neuro. Oncol. 14, 315-325 https://doi.org/10.1093/neuonc/nor209

Wang Y, Zhang X, Tang W, Lin Z, Xu L, Dong R, Li Y, Li J, Zhang Z, Li X, et al. (2017): miR-130a upregulates mTOR pathway by targeting TSC1 and is transactivated by NF- $\mathrm{KB}$ in high-grade serous ovarian carcinoma. Cell Death Differ. 24, 2089-2100 https://doi.org/10.1038/cdd.2017.129

Wang J, Qi Q, Zhou W, Feng Z, Huang B, Chen A, Zhang D, Li W, Zhang Q, Jiang Z, et al. (2018): Inhibition of glioma growth by flavokawain $B$ is mediated through endoplasmic reticulum stress induced autophagy. Autophagy 14, 2007-2022 https://doi.org/10.1080/15548627.2018.1501133

Wang J, Ma J (2019): Integrated transcriptomic analysis of necrosisrelated gene in diffuse gliomas. J. Neurol. Surg. A Cent. Eur. Neurosurg. 80, 240-249 https://doi.org/10.1055/s-0039-1683448

Wang Y, Liu X, Guan G, Xiao Z, Zhao W, Zhuang M (2019a): Identification of a five-pseudogene signature for predicting survival and its ceRNA network in glioma. Front. Oncol. 9, 1059 https://doi.org/10.3389/fonc.2019.01059

Wang Y, Liu X, Guan G, Zhao W, Zhuang M (2019b): A risk classification system with five-gene for survival prediction of glioblastoma patients. Front. Neurol. 10, 745 https://doi.org/10.3389/fneur.2019.00745

Wang Z, Gao L, Guo X, Feng C, Lian W, Deng K, Xing B (2019c): Development and validation of a nomogram with an autophagy-related gene signature for predicting survival in patients with glioblastoma. Aging 11, 12246-12269 https://doi.org/10.18632/aging.102566

Wang P, Zeng Z, Shen X, Tian X, Ye Q (2020a): Identification of a multi-RNA-type-based signature for recurrence-free survival prediction in patients with uterine corpus endometrial carcinoma. DNA Cell Biol. 39, 615-630 https://doi.org/10.1089/dna.2019.5148

Wang QW, Liu HJ, Zhao Z, Zhang Y, Wang Z, Jiang T, Bao ZS (2020b): Prognostic correlation of autophagy-related gene expression-based risk signature in patients with glioblastoma. Onco. Targets Ther. 13, 95-107 https://doi.org/10.2147/OTT.S238332

Wang Y, Zhao W, Xiao Z, Guan G, Liu X, Zhuang M (2020c): A risk signature with four autophagy-related genes for predicting survival of glioblastoma multiforme. J. Cell Mol. Med. 24, 3807-3821

https://doi.org/10.1111/jcmm.14938

Wang C, Qiu J, Chen S, Li Y, Hu H, Cai Y, Hou L (2021): Prognostic model and nomogram construction based on autophagy signatures in lower grade glioma. J. Cell Physiol. 236, 235-248 https://doi.org/10.1002/jcp.29837

Wu H, Zhu H, Liu DX, Niu TK, Ren X, Patel R, Hait WN, Yang JM (2009): Silencing of elongation factor-2 kinase potentiates the effect of 2-deoxy-D-glucose against human glioma cells through blunting of autophagy. Cancer Res. 69, 2453-2460 https://doi.org/10.1158/0008-5472.CAN-08-2872

Wu F, Zhao Z, Chai R, Liu Y, Wang K, Wang Z, Li G, Huang R, Jiang H, Zhang K (2018): Expression profile analysis of antisense long non-coding RNA identifies WDFY3-AS2 as a prognostic biomarker in diffuse glioma. Cancer Cell Int. 18, 107 https://doi.org/10.1186/s12935-018-0603-2

Wu F, Zhao Z, Chai RC, Liu YQ, Li GZ, Jiang HY, Jiang T (2019): Prognostic power of a lipid metabolism gene panel for diffuse gliomas. J. Cell Mol. Med. 23, 7741-7748 https://doi.org/10.1111/jcmm.14647

Yang TQ, Lu XJ, Wu TF, Ding DD, Zhao ZH, Chen GL, Xie XS, Li B, Wei YX, Guo LC, et al. (2014): MicroRNA-16 inhibits glioma cell growth and invasion through suppression of BCL2 and the nuclear factor-kB1/MMP9 signaling pathway. Cancer Sci. 105, 265-271

https://doi.org/10.1111/cas.12351

Yang N, Wang P, Wang WJ, Song YZ, Liang ZQ (2015): Inhibition of cathepsin $\mathrm{L}$ sensitizes human glioma cells to ionizing radiation in vitro through NF- $\mathrm{kB}$ signaling pathway. Acta Pharmacol. Sin. 36, 400-410 https://doi.org/10.1038/aps.2014.148

Yang K, Niu L, Bai Y, Le W (2019): Glioblastoma: Targeting the autophagy in tumorigenesis. Brain Res. Bull. 153, 334-340 https://doi.org/10.1016/j.brainresbull.2019.09.012 
Zhang L, Zhang Y, Liu XY, Qin ZH, Yang JM (2011): Expression of elongation factor-2 kinase contributes to anoikis resistance and invasion of human glioma cells. Acta Pharmacol. Sin. 32, 361-367 https://doi.org/10.1038/aps.2010.213

Zhang X, Wang X, Xu S, Li X, Ma X (2018): Cathepsin B contributes to radioresistance by enhancing homologous recombination in glioblastoma. Biomed. Pharmacother. 107, 390-396 https://doi.org/10.1016/j.biopha.2018.08.007

Zhang GH, Zhong QY, Gou XX, Fan EX, Shuai Y, Wu MN, Yue G) (2019): Seven genes for the prognostic prediction in patients with glioma. Clin. Transl. Oncol. 21, 1327-1335 https://doi.org/10.1007/s12094-019-02057-3
Zhang Y, Liu Y, Liu H, Zhao Z, Wu F, Zeng F (2020): Clinical and biological significances of a methyltransferase-related signature in diffuse glioma. Front. Oncol. 10, 508 https://doi.org/10.3389/fonc.2020.00508

Zhu WL, Tong H, Teh JT, Wang M (2014): Forkhead box protein O3 transcription factor negatively regulates autophagy in human cancer cells by inhibiting forkhead box protein $\mathrm{O} 1$ expression and cytosolic accumulation. Plos One 9, e115087 https://doi.org/10.1371/journal.pone.0115087

Received: September 23, 2020

Final version accepted: March 22, 2021 
Supplementary Material

\title{
Identification of an autophagy-related 10-IncRNA-mRNA signature for distinguishing glioblastoma multiforme from lower-grade glioma and prognosis prediction
}

\author{
Bo Wei ${ }^{1, *}$, Le Wang ${ }^{2, *}$ and Jingwei Zhao ${ }^{1}$ \\ ${ }^{1}$ Department of Neurosurgery, China-Japan Union Hospital of Jilin University, Changchun, Jilin, China \\ 2 Department of Ophthalmology, The First Hospital of Jilin University, Changchun, Jilin, China
}

Supplementary table S1. The sample ID of TCGA and CGGA

\begin{tabular}{ll}
\hline Symbol & Symbol \\
\hline CGGA_1473 & TCGA-26-1442-01 \\
CGGA_1443 & TCGA-06-0129-01 \\
CGGA_1065 & TCGA-19-5960-01 \\
CGGA_1003 & TCGA-32-1980-01 \\
CGGA_1744 & TCGA-19-1390-01 \\
CGGA_1649 & TCGA-06-5417-01 \\
CGGA_1903 & TCGA-19-2620-01 \\
CGGA_P492 & TCGA-32-1982-01 \\
CGGA_P183 & TCGA-06-0132-01 \\
CGGA_1158 & TCGA-06-0747-01 \\
CGGA_1239 & TCGA-27-1830-01 \\
CGGA_1675 & TCGA-28-5216-01 \\
CGGA_1627 & TCGA-41-3915-01 \\
CGGA_P177 & TCGA-06-0219-01 \\
CGGA_42 & TCGA-32-1970-01 \\
CGGA_1309 & TCGA-06-5411-01 \\
CGGA_P131 & TCGA-27-2523-01 \\
CGGA_1211 & TCGA-06-2570-01 \\
CGGA_336 & TCGA-06-5416-01 \\
CGGA_1400 & TCGA-06-0743-01 \\
CGGA_810 & TCGA-41-4097-01 \\
CGGA_1562 & TCGA-19-2624-01 \\
CGGA_50 & TCGA-06-0882-01 \\
CGGA_P176 & TCGA-02-0047-01 \\
CGGA_P415 & TCGA-06-1804-01 \\
CGGA_P500 & TCGA-32-4213-01 \\
CGGA_P585 & TCGA-06-2559-01 \\
CGGA_1526 & TCGA-06-0745-01 \\
CGGA_320 & TCGA-06-0878-01 \\
CGGA_P7 & TCGA-06-0178-01 \\
CGGA_707 & TCGA-14-1829-01 \\
CGGA_P132 & TCGA-12-5295-01 \\
\hline
\end{tabular}

\begin{tabular}{ll}
\hline Symbol & Symbol \\
\hline CGGA_1386 & TCGA-16-0846-01 \\
CGGA_P156 & TCGA-26-5134-01 \\
CGGA_P399 & TCGA-19-2625-01 \\
CGGA_1208 & TCGA-06-0238-01 \\
CGGA_1595 & TCGA-06-0750-01 \\
CGGA_P17 & TCGA-41-2571-01 \\
CGGA_290 & TCGA-32-2615-01 \\
CGGA_1415 & TCGA-06-0649-01 \\
CGGA_1642 & TCGA-06-5856-01 \\
CGGA_1695 & TCGA-06-2561-01 \\
CGGA_903 & TCGA-06-0645-01 \\
CGGA_P122 & TCGA-06-2565-01 \\
CGGA_1551 & TCGA-76-4926-01 \\
CGGA_D28 & TCGA-06-0139-01 \\
CGGA_1528 & TCGA-06-0125-01 \\
CGGA_103 & TCGA-15-1444-01 \\
CGGA_1413 & TCGA-12-1597-01 \\
CGGA_1383 & TCGA-26-5139-01 \\
CGGA_P163 & TCGA-06-0749-01 \\
CGGA_869 & TCGA-06-0744-01 \\
CGGA_1232 & TCGA-27-1837-01 \\
CGGA_P596 & TCGA-12-0619-01 \\
CGGA_P18 & TCGA-26-5132-01 \\
CGGA_P392 & TCGA-26-5136-01 \\
CGGA_1192 & TCGA-27-1835-01 \\
CGGA_2047 & TCGA-06-5418-01 \\
CGGA_P401 & TCGA-14-0790-01 \\
CGGA_1273 & TCGA-06-0156-01 \\
CGGA_1164 & TCGA-28-2514-01 \\
CGGA_1368 & TCGA-06-0158-01 \\
CGGA_1156 & TCGA-06-0141-01 \\
CGGA_1653 & TCGA-06-0211-01 \\
CGGA_1706 & TCGA-32-2638-01 \\
CGGA_1530 & TCGA-02-2485-01 \\
\hline &
\end{tabular}

\begin{tabular}{ll}
\hline Symbol & Symbol \\
\hline CGGA_1634 & TCGA-06-2557-01 \\
CGGA_1502 & TCGA-06-5410-01 \\
CGGA_1559 & TCGA-02-2483-01 \\
CGGA_P520 & TCGA-28-174-01 \\
CGGA_1617 & TCGA-12-3653-01 \\
CGGA_362 & TCGA-16-1045-01 \\
CGGA_890 & TCGA-06-2567-01 \\
CGGA_1604 & TCGA-28-2513-01 \\
CGGA_1516 & TCGA-06-0686-01 \\
CGGA_P13 & TCGA-12-0618-01 \\
CGGA_1647 & TCGA-06-5858-01 \\
CGGA_1863 & TCGA-28-5218-01 \\
CGGA_1517 & TCGA-06-0138-01 \\
CGGA_1640 & TCGA-06-0210-01 \\
CGGA_1319 & TCGA-06-5413-01 \\
CGGA_P150 & TCGA-28-1753-01 \\
CGGA_2081 & TCGA-12-0821-01 \\
CGGA_1127 & TCGA-06-0130-01 \\
CGGA_P468 & TCGA-06-0190-01 \\
CGGA_157 & TCGA-27-2528-01 \\
CGGA_554 & TCGA-41-2572-01 \\
CGGA_1264 & TCGA-41-5651-01 \\
CGGA_P111 & TCGA-19-2629-01 \\
CGGA_P421 & TCGA-76-4925-01 \\
CGGA_1667 & TCGA-08-0386-01 \\
CGGA_P84 & TCGA-14-0789-01 \\
CGGA_1369 & TCGA-12-3652-01 \\
CGGA_1701 & TCGA-27-1834-01 \\
CGGA_1554 & TCGA-12-3650-01 \\
CGGA_1592 & TCGA-26-5135-01 \\
CGGA_P416 & TCGA-19-2619-01 \\
CGGA_P174 & TCGA-06-0157-01 \\
CGGA_P146 & TCGA-28-5215-01 \\
CGGA_P422 & TCGA-27-2519-01 \\
\hline &
\end{tabular}


Supplementary table S1. The sample ID of TCGA and CGGA (continued)

\begin{tabular}{|c|c|}
\hline Symbol & Symbol \\
\hline CGGA_1103 & TCGA-28-5204-01 \\
\hline CGGA_P270 & TCGA-14-0781-01 \\
\hline CGGA_1720 & TCGA-06-0187-01 \\
\hline CGGA_1147 & TCGA-06-0174-01 \\
\hline CGGA_1704 & TCGA-76-4927-01 \\
\hline CGGA_1235 & TCGA-06-2562-01 \\
\hline CGGA_1643 & TCGA-02-0055-01 \\
\hline CGGA_1504 & TCGA-76-4928-01 \\
\hline CGGA_492 & TCGA-27-2521-01 \\
\hline CGGA_1569 & TCGA-06-5414-01 \\
\hline CGGA_1679 & TCGA-32-2616-01 \\
\hline CGGA_1513 & TCGA-14-0871-01 \\
\hline CGGA_1786 & TCGA-76-4931-01 \\
\hline CGGA_P446 & TCGA-32-5222-01 \\
\hline CGGA_1747 & TCGA-19-4065-01 \\
\hline CGGA_809 & TCGA-06-5412-01 \\
\hline CGGA_P143 & TCGA-06-5859-01 \\
\hline CGGA_1534 & TCGA-28-5209-01 \\
\hline CGGA_1737 & TCGA-15-0742-01 \\
\hline CGGA_1661 & TCGA-12-5299-01 \\
\hline CGGA_799 & TCGA-32-2634-01 \\
\hline CGGA_P364 & TCGA-14-2554-01 \\
\hline CGGA_1536 & TCGA-14-1823-01 \\
\hline CGGA_1641 & TCGA-06-0168-01 \\
\hline CGGA_P16 & TCGA-27-1832-01 \\
\hline CGGA_P314 & TCGA-28-5207-01 \\
\hline CGGA_1169 & TCGA-28-2509-01 \\
\hline CGGA_1087 & TCGA-14-0817-01 \\
\hline CGGA_1723 & TCGA-06-2563-01 \\
\hline CGGA_265 & TCGA-06-2564-01 \\
\hline CGGA_1433 & TCGA-14-1825-01 \\
\hline CGGA_P137 & TCGA-06-2569-01 \\
\hline CGGA_P142 & TCGA-26-5133-01 \\
\hline CGGA_1377 & TCGA-12-0616-01 \\
\hline CGGA_1305 & TCGA-27-2524-01 \\
\hline CGGA_1566 & TCGA-06-0184-01 \\
\hline CGGA_1362 & TCGA-06-2558-01 \\
\hline CGGA_1557 & TCGA-28-5208-01 \\
\hline CGGA_D50 & TCGA-19-1787-01 \\
\hline CGGA_1401 & TCGA-27-2526-01 \\
\hline CGGA_1463 & TCGA-02-2486-01 \\
\hline CGGA_1552 & TCGA-14-1034-01 \\
\hline CGGA_1121 & TCGA-27-1831-01 \\
\hline CGGA_165 & TCGA-28-5220-01 \\
\hline CGGA_P633 & TCGA-32-2632-01 \\
\hline CGGA_P356 & TCGA-76-4932-01 \\
\hline CGGA_P103 & TCGA-06-5408-01 \\
\hline CGGA_1882 & TCGA-76-4929-01 \\
\hline CGGA_2079 & TCGA-06-0644-01 \\
\hline
\end{tabular}

\begin{tabular}{|c|c|}
\hline Symbol & Symbol \\
\hline CGGA_1546 & TCGA-14-0787-01 \\
\hline CGGA_1183 & TCGA-HT-A74L-01 \\
\hline CGGA_1407 & TCGA-FG-A4MX-01 \\
\hline CGGA_1644 & TCGA-P5-A5F6-01 \\
\hline CGGA_1721 & TCGA-HT-7873-01 \\
\hline CGGA_135 & TCGA-S9-A89V-01 \\
\hline CGGA_P109 & TCGA-FG-8186-01 \\
\hline CGGA_1689 & TCGA-HT-7884-01 \\
\hline CGGA_1523 & TCGA-E1-A7YW-01 \\
\hline CGGA_P173 & TCGA-DB-A64R-01 \\
\hline CGGA_1152 & TCGA-HT-7692-01 \\
\hline CGGA_P144 & TCGA-E1-5318-01 \\
\hline CGGA_1037 & TCGA-E1-A7YO-01 \\
\hline CGGA_1657 & TCGA-HT-8564-01 \\
\hline CGGA_1630 & TCGA-E1-A7YH-01 \\
\hline CGGA_1539 & TCGA-FG-8185-01 \\
\hline CGGA_1488 & TCGA-TQ-A7RG-01 \\
\hline CGGA_1658 & TCGA-E1-A7YV-01 \\
\hline CGGA_1533 & TCGA-S9-A7IY-01 \\
\hline CGGA_P15 & TCGA-HT-8114-01 \\
\hline CGGA_901 & TCGA-CS-6667-01 \\
\hline CGGA_P269 & TCGA-RY-A840-01 \\
\hline CGGA_1181 & TCGA-DH-A66F-01 \\
\hline CGGA_P286 & TCGA-QH-A6X4-01 \\
\hline CGGA_1568 & TCGA-TQ-A7RO-01 \\
\hline CGGA_634 & TCGA-P5-A781-01 \\
\hline CGGA_P27 & TCGA-HT-7693-01 \\
\hline CGGA_1728 & TCGA-RY-A845-01 \\
\hline CGGA_1985 & TCGA-S9-A7QZ-01 \\
\hline CGGA_1303 & TCGA-TQ-A7RQ-01 \\
\hline CGGA_1256 & TCGA-HT-7855-01 \\
\hline CGGA_1416 & TCGA-TQ-A7RI-01 \\
\hline CGGA_2075 & TCGA-HT-8012-01 \\
\hline CGGA_1731 & TCGA-TQ-A7RR-01 \\
\hline CGGA_625 & TCGA-HT-7480-01 \\
\hline CGGA_1212 & TCGA-DU-A5TW-01 \\
\hline CGGA_1269 & TCGA-S9-A7QY-01 \\
\hline CGGA_P604 & TCGA-DH-A7UV-01 \\
\hline CGGA_1946 & TCGA-S9-A7J2-01 \\
\hline CGGA_1814 & TCGA-TQ-A8XE-01 \\
\hline CGGA_P508 & TCGA-DB-A64S-01 \\
\hline CGGA_P298 & TCGA-WY-A85D-01 \\
\hline CGGA_P110 & TCGA-QH-A6CW-01 \\
\hline CGGA_1575 & TCGA-HT-7877-01 \\
\hline CGGA_1819 & TCGA-P5-A5F0-01 \\
\hline CGGA_288 & TCGA-HT-7483-01 \\
\hline CGGA_1291 & TCGA-HT-8108-01 \\
\hline CGGA_P93 & TCGA-HT-7874-01 \\
\hline CGGA_1482 & TCGA-DB-A4XC-01 \\
\hline
\end{tabular}

\begin{tabular}{|c|c|}
\hline Symbol & Symbol \\
\hline CGGA_108 & TCGA-S9-A7J1-01 \\
\hline CGGA_P311 & TCGA-F6-A8O3-01 \\
\hline CGGA_P182 & TCGA-S9-A7R1-01 \\
\hline CGGA_1191 & TCGA-HT-7469-01 \\
\hline CGGA_1655 & TCGA-HT-7875-01 \\
\hline CGGA_P337 & TCGA-E1-A7YU-01 \\
\hline CGGA_1345 & TCGA-QH-A6CZ-01 \\
\hline CGGA_1663 & TCGA-FG-7641-01 \\
\hline CGGA_1496 & TCGA-CS-5393-01 \\
\hline CGGA_1392 & TCGA-TQ-A7RN-01 \\
\hline CGGA_663 & TCGA-CS-5394-01 \\
\hline CGGA_1875 & TCGA-FG-8182-01 \\
\hline CGGA_194 & TCGA-CS-6670-01 \\
\hline CGGA_P512 & TCGA-S9-A6TZ-01 \\
\hline CGGA_881 & TCGA-DB-5275-01 \\
\hline CGGA_1472 & TCGA-DU-6396-01 \\
\hline CGGA_1404 & TCGA-FG-A60K-01 \\
\hline CGGA_1510 & TCGA-DB-A64U-01 \\
\hline CGGA_1771 & TCGA-HW-7491-01 \\
\hline CGGA_1912 & TCGA-RY-A83X-01 \\
\hline CGGA_1134 & TCGA-HT-8107-01 \\
\hline CGGA_1238 & TCGA-DB-A64Q-01 \\
\hline CGGA_1402 & TCGA-E1-5304-01 \\
\hline CGGA_1524 & TCGA-TQ-A7RV-01 \\
\hline CGGA_1718 & TCGA-HW-8320-01 \\
\hline CGGA_1580 & TCGA-R8-A6MK-01 \\
\hline CGGA_1899 & TCGA-DU-7007-01 \\
\hline CGGA_1389 & TCGA-HT-8113-01 \\
\hline CGGA_1714 & TCGA-DU-7298-01 \\
\hline CGGA_P147 & TCGA-VW-A7QS-01 \\
\hline CGGA_P328 & TCGA-DH-5141-01 \\
\hline CGGA_1659 & TCGA-S9-A7R8-01 \\
\hline CGGA_1586 & TCGA-QH-A86X-01 \\
\hline CGGA_1286 & TCGA-QH-A870-01 \\
\hline CGGA_1715 & TCGA-HT-7688-01 \\
\hline CGGA_1587 & TCGA-DB-A75L-01 \\
\hline CGGA_1427 & TCGA-S9-A6WO-01 \\
\hline CGGA_P19 & TCGA-TM-A84Q-01 \\
\hline CGGA_1626 & TCGA-S9-A7QW-01 \\
\hline CGGA_279 & TCGA-HT-7468-01 \\
\hline CGGA_P437 & TCGA-DU-8164-01 \\
\hline CGGA_1571 & TCGA-HW-7487-01 \\
\hline CGGA_1155 & TCGA-DB-A4XG-01 \\
\hline CGGA_P411 & TCGA-DH-A66G-01 \\
\hline CGGA_1318 & TCGA-HW-8321-01 \\
\hline CGGA_1066 & TCGA-P5-A77X-01 \\
\hline CGGA_1440 & TCGA-DU-A6S2-01 \\
\hline CGGA_724 & TCGA-TM-A84L-01 \\
\hline CGGA_1260 & TCGA-VV-A829-01 \\
\hline
\end{tabular}


Supplementary table S1. The sample ID of TCGA and CGGA (continued)

\begin{tabular}{|c|c|}
\hline Symbol & Symbol \\
\hline CGGA_1471 & TCGA-DH-A7UT-01 \\
\hline CGGA_P310 & TCGA-DU-A7TG-01 \\
\hline CGGA_1339 & TCGA-DU-A6S6-01 \\
\hline CGGA_1880 & TCGA-HT-7879-01 \\
\hline CGGA_1131 & TCGA-DB-A4XD-01 \\
\hline CGGA_P438 & TCGA-S9-A6TV-01 \\
\hline CGGA_1321 & TCGA-DU-5849-01 \\
\hline CGGA_1162 & TCGA-S9-A6TX-01 \\
\hline CGGA_1097 & TCGA-S9-A7J3-01 \\
\hline CGGA_P179 & TCGA-DU-5870-01 \\
\hline CGGA_1716 & TCGA-HT-7681-01 \\
\hline CGGA_P28 & TCGA-FG-7637-01 \\
\hline CGGA_1431 & TCGA-HT-A618-01 \\
\hline CGGA_1620 & TCGA-HW-8319-01 \\
\hline CGGA_P505 & TCGA-DU-A76O-01 \\
\hline CGGA_P568 & TCGA-HT-A61B-01 \\
\hline CGGA_1703 & TCGA-WY-A85A-01 \\
\hline CGGA_1725 & TCGA-TM-A84R-01 \\
\hline CGGA_621 & TCGA-DU-A5TS-01 \\
\hline CGGA_1955 & TCGA-FG-8189-01 \\
\hline CGGA_P5 & TCGA-TQ-A7RH-01 \\
\hline CGGA_1594 & TCGA-DU-8166-01 \\
\hline CGGA_1161 & TCGA-HW-7495-01 \\
\hline CGGA_1041 & TCGA-S9-A7R7-01 \\
\hline CGGA_1361 & TCGA-RY-A83Y-01 \\
\hline CGGA_1688 & TCGA-E1-5305-01 \\
\hline CGGA_1994 & TCGA-VM-A8C8-01 \\
\hline CGGA_P439 & TCGA-DH-A66B-01 \\
\hline CGGA_1501 & TCGA-DU-8168-01 \\
\hline CGGA_721 & TCGA-DU-7009-01 \\
\hline CGGA_1036 & TCGA-E1-A7Z6-01 \\
\hline CGGA_1525 & TCGA-TM-A84H-01 \\
\hline CGGA_1662 & TCGA-S9-A6U8-01 \\
\hline CGGA_509 & TCGA-W9-A837-01 \\
\hline CGGA_1452 & TCGA-P5-A5F4-01 \\
\hline CGGA_1441 & TCGA-TQ-A7RV-02 \\
\hline CGGA_1902 & TCGA-P5-A730-01 \\
\hline CGGA_P199 & TCGA-HT-7610-01 \\
\hline CGGA_1648 & TCGA-P5-A780-01 \\
\hline CGGA_P151 & TCGA-DU-A76R-01 \\
\hline CGGA_1207 & TCGA-S9-A7IQ-01 \\
\hline CGGA_1690 & TCGA-VM-A8CE-01 \\
\hline CGGA_P145 & TCGA-DB-A75M-01 \\
\hline CGGA_1735 & TCGA-S9-A6U2-01 \\
\hline CGGA_1739 & TCGA-HT-7856-01 \\
\hline CGGA_P155 & TCGA-HT-8109-01 \\
\hline CGGA_106 & TCGA-CS-6666-01 \\
\hline CGGA_1508 & TCGA-DH-A7US-01 \\
\hline CGGA_1671 & TCGA-RY-A843-01 \\
\hline
\end{tabular}

\begin{tabular}{|c|c|}
\hline Symbol & Symbol \\
\hline CGGA_P315 & TCGA-VM-A8CB-01 \\
\hline CGGA_P181 & TCGA-HT-7482-01 \\
\hline CGGA_P501 & TCGA-HT-7477-01 \\
\hline CGGA_P358 & TCGA-HT-7609-01 \\
\hline CGGA_1417 & TCGA-DB-A75K-01 \\
\hline CGGA_882 & TCGA-VM-A8CF-01 \\
\hline CGGA_P335 & TCGA-FN-7833-01 \\
\hline CGGA_1018 & TCGA-E1-5307-01 \\
\hline CGGA_1850 & TCGA-HT-7478-01 \\
\hline CGGA_1736 & TCGA-E1-A7YE-01 \\
\hline CGGA_1398 & TCGA-DU-A7T8-01 \\
\hline CGGA_1178 & TCGA-E1-A7YS-01 \\
\hline CGGA_1857 & TCGA-HT-7881-01 \\
\hline CGGA_P346 & TCGA-DU-6542-01 \\
\hline CGGA_1527 & TCGA-DU-7302-01 \\
\hline CGGA_1618 & TCGA-DB-A4XF-01 \\
\hline CGGA_1492 & TCGA-HT-8111-01 \\
\hline CGGA_1901 & TCGA-HW-A5KJ-01 \\
\hline CGGA_1906 & TCGA-DU-6408-01 \\
\hline CGGA_1487 & TCGA-E1-5319-01 \\
\hline CGGA_1623 & TCGA-S9-A6TS-01 \\
\hline CGGA_P185 & TCGA-P5-A5EV-01 \\
\hline CGGA_1598 & TCGA-FG-5965-01 \\
\hline CGGA_1686 & TCGA-FG-A4MY-01 \\
\hline CGGA_P31 & TCGA-FG-A70Y-01 \\
\hline CGGA_1854 & TCGA-TQ-A7RW-01 \\
\hline CGGA_1410 & TCGA-DU-A7TA-01 \\
\hline CGGA_1645 & TCGA-TM-A84T-01 \\
\hline CGGA_P483 & TCGA-E1-A7Z4-01 \\
\hline CGGA_P21 & TCGA-HT-7880-01 \\
\hline CGGA_1058 & TCGA-WY-A85B-01 \\
\hline CGGA_1565 & TCGA-FG-6689-01 \\
\hline CGGA_1669 & TCGA-HT-A5RB-01 \\
\hline CGGA_P83 & TCGA-FG-8187-01 \\
\hline CGGA_P316 & TCGA-TM-A84I-01 \\
\hline CGGA_1700 & TCGA-P5-A5EZ-01 \\
\hline CGGA_1335 & TCGA-FG-6690-01 \\
\hline CGGA_1591 & TCGA-HT-7472-01 \\
\hline CGGA_730 & TCGA-S9-A6U6-01 \\
\hline CGGA_1055 & TCGA-QH-A6CY-01 \\
\hline CGGA_P20 & TCGA-DU-6397-01 \\
\hline CGGA_1543 & TCGA-EZ-7264-01 \\
\hline CGGA_867 & TCGA-QH-A65V-01 \\
\hline CGGA_1535 & TCGA-S9-A6U9-01 \\
\hline CGGA_1184 & TCGA-TQ-A7RJ-01 \\
\hline CGGA_1727 & TCGA-VM-A8CH-01 \\
\hline CGGA_1673 & TCGA-DB-A4XB-01 \\
\hline CGGA_1132 & TCGA-HT-7677-01 \\
\hline CGGA_P106 & TCGA-R8-A6ML-01 \\
\hline
\end{tabular}

\begin{tabular}{|c|c|}
\hline Symbol & Symbol \\
\hline CGGA_1126 & TCGA-E1-A7YM-01 \\
\hline CGGA_457 & TCGA-P5-A72X-01 \\
\hline CGGA_1505 & TCGA-HT-7690-01 \\
\hline CGGA_1477 & TCGA-DB-A64X-01 \\
\hline CGGA_521 & TCGA-TQ-A7RF-01 \\
\hline CGGA_1589 & TCGA-HT-7604-01 \\
\hline CGGA_P385 & TCGA-HW-A5KL-01 \\
\hline CGGA_P510 & TCGA-DB-5270-01 \\
\hline CGGA_1033 & TCGA-WY-A858-01 \\
\hline CGGA_1159 & TCGA-IK-8125-01 \\
\hline CGGA_1451 & TCGA-CS-6669-01 \\
\hline CGGA_888 & TCGA-S9-A6WD-01 \\
\hline CGGA_1826 & TCGA-E1-5311-01 \\
\hline CGGA_P279 & TCGA-TM-A84S-01 \\
\hline CGGA_1693 & TCGA-HT-7473-01 \\
\hline CGGA_1017 & TCGA-DU-7309-01 \\
\hline CGGA_P388 & TCGA-HT-7694-01 \\
\hline CGGA_P98 & TCGA-IK-7675-01 \\
\hline CGGA_1750 & TCGA-TM-A84G-01 \\
\hline CGGA_1455 & TCGA-TM-A7CF-01 \\
\hline CGGA_1434 & TCGA-S9-A7IZ-01 \\
\hline CGGA_1579 & TCGA-DU-A5TR-01 \\
\hline CGGA_705 & TCGA-HT-A615-01 \\
\hline CGGA_2046 & TCGA-HT-8013-01 \\
\hline CGGA_530 & TCGA-WY-A859-01 \\
\hline CGGA_1597 & TCGA-P5-A5F2-01 \\
\hline CGGA_1371 & TCGA-TM-A84M-01 \\
\hline CGGA_1425 & TCGA-WY-A85C-01 \\
\hline CGGA_1421 & TCGA-HT-A5R7-01 \\
\hline CGGA_1403 & TCGA-E1-5322-01 \\
\hline CGGA_482 & TCGA-HT-7858-01 \\
\hline CGGA_P102 & TCGA-R8-A73M-01 \\
\hline CGGA_1608 & TCGA-DH-A66D-01 \\
\hline CGGA_1057 & TCGA-DU-6400-01 \\
\hline CGGA_P157 & TCGA-HT-A5R5-01 \\
\hline CGGA_1866 & TCGA-S9-A6U1-01 \\
\hline CGGA_503 & TCGA-DU-7304-01 \\
\hline CGGA_1106 & TCGA-QH-A65Z-01 \\
\hline CGGA_1601 & TCGA-FG-A710-01 \\
\hline CGGA_P326 & TCGA-DU-5871-01 \\
\hline CGGA_P99 & TCGA-HT-7481-01 \\
\hline CGGA_1664 & TCGA-S9-A6TY-01 \\
\hline CGGA_1108 & TCGA-DU-8163-01 \\
\hline CGGA_2008 & TCGA-HT-7689-01 \\
\hline CGGA_1458 & TCGA-HT-8105-01 \\
\hline CGGA_2002 & TCGA-S9-A6WH-01 \\
\hline CGGA_P121 & TCGA-DU-A7TI-01 \\
\hline CGGA_1606 & TCGA-HT-A61A-01 \\
\hline CGGA_862 & TCGA-HT-7474-01 \\
\hline
\end{tabular}


Supplementary table S1. The sample ID of TCGA and CGGA (continued)

\begin{tabular}{|c|c|}
\hline Symbol & Symbol \\
\hline CGGA_2078 & TCGA-FG-7634-01 \\
\hline CGGA_1420 & TCGA-DU-7019-01 \\
\hline CGGA_1687 & TCGA-DB-5281-01 \\
\hline CGGA_1447 & TCGA-HT-7601-01 \\
\hline CGGA_1621 & TCGA-FG-8181-01 \\
\hline CGGA_1583 & TCGA-VM-A8CA-01 \\
\hline CGGA_1680 & TCGA-E1-A7YY-01 \\
\hline CGGA_1048 & TCGA-E1-A7YK-01 \\
\hline CGGA_2006 & TCGA-QH-A6X9-01 \\
\hline CGGA_1811 & TCGA-S9-A6WI-01 \\
\hline CGGA_1148 & TCGA-FG-8188-01 \\
\hline CGGA_703 & TCGA-HT-8019-01 \\
\hline CGGA_1300 & TCGA-E1-A7Z3-01 \\
\hline CGGA_107 & TCGA-S9-A6WE-01 \\
\hline CGGA_P619 & TCGA-HW-8322-01 \\
\hline CGGA_1317 & TCGA-P5-A731-01 \\
\hline CGGA_1650 & TCGA-DU-7015-01 \\
\hline CGGA_1467 & TCGA-TM-A7CA-01 \\
\hline CGGA_1764 & TCGA-DH-5143-01 \\
\hline CGGA_652 & TCGA-DU-6401-01 \\
\hline CGGA_1605 & TCGA-FG-6691-01 \\
\hline CGGA_1154 & TCGA-P5-A5F1-01 \\
\hline CGGA_P295 & TCGA-TM-A7CF-02 \\
\hline CGGA_1785 & TCGA-S9-A7R3-01 \\
\hline CGGA_1135 & TCGA-QH-A6CU-01 \\
\hline CGGA_1612 & TCGA-DU-7011-01 \\
\hline CGGA_1157 & TCGA-S9-A6TW-01 \\
\hline CGGA_1195 & TCGA-DU-A7TC-01 \\
\hline CGGA_1776 & TCGA-DB-A4X9-01 \\
\hline CGGA_2129 & TCGA-HT-8010-01 \\
\hline CGGA_1567 & TCGA-KT-A74X-01 \\
\hline CGGA_112 & TCGA-TQ-A7RK-02 \\
\hline CGGA_P160 & TCGA-DB-5279-01 \\
\hline CGGA_1972 & TCGA-QH-A6X8-01 \\
\hline CGGA_1012 & TCGA-DU-A5TU-01 \\
\hline CGGA_1356 & TCGA-S9-A6U5-01 \\
\hline CGGA_1560 & TCGA-HT-7605-01 \\
\hline CGGA_1204 & TCGA-HT-A74J-01 \\
\hline CGGA_P594 & TCGA-RY-A847-01 \\
\hline CGGA_889 & TCGA-DU-A6S7-01 \\
\hline CGGA_D19 & TCGA-P5-A737-01 \\
\hline CGGA_1435 & TCGA-HT-7611-01 \\
\hline CGGA_1111 & TCGA-DU-5855-01 \\
\hline CGGA_1334 & TCGA-DU-7300-01 \\
\hline CGGA_1769 & TCGA-DB-5280-01 \\
\hline CGGA_1660 & TCGA-DB-A4XE-01 \\
\hline CGGA_1223 & TCGA-VV-A86M-01 \\
\hline CGGA_P625 & TCGA-DB-A64L-01 \\
\hline CGGA_1738 & TCGA-TM-A84O-01 \\
\hline
\end{tabular}

\begin{tabular}{|c|c|}
\hline Symbol & Symbol \\
\hline CGGA_P153 & TCGA-DU-7008-01 \\
\hline CGGA_1614 & TCGA-P5-A5EX-01 \\
\hline CGGA_P11 & TCGA-QH-A65S-01 \\
\hline CGGA_1840 & TCGA-HT-7695-01 \\
\hline CGGA_780 & TCGA-HT-7854-01 \\
\hline CGGA_P89 & TCGA-CS-4944-01 \\
\hline CGGA_1833 & TCGA-DU-5870-02 \\
\hline CGGA_J73 & TCGA-FG-8191-01 \\
\hline CGGA_1295 & TCGA-TM-A7C4-01 \\
\hline CGGA_P128 & TCGA-DB-A64P-01 \\
\hline CGGA_1563 & TCGA-P5-A5EW-01 \\
\hline CGGA_1541 & TCGA-S9-A7QX-01 \\
\hline CGGA_1350 & TCGA-S9-A6WQ-01 \\
\hline CGGA_861 & TCGA-QH-A65R-01 \\
\hline CGGA_1697 & TCGA-HT-7607-01 \\
\hline CGGA_1032 & TCGA-DB-A4XA-01 \\
\hline CGGA_1507 & TCGA-HT-A4DV-01 \\
\hline CGGA_1953 & TCGA-DB-A64V-01 \\
\hline CGGA_1426 & TCGA-HT-7620-01 \\
\hline CGGA_P112 & TCGA-QH-A6XA-01 \\
\hline CGGA_1740 & TCGA-FG-5962-01 \\
\hline CGGA_P30 & TCGA-S9-A6WN-01 \\
\hline CGGA_1685 & TCGA-HT-7475-01 \\
\hline CGGA_1613 & TCGA-HT-7470-01 \\
\hline CGGA_1337 & TCGA-HT-8558-01 \\
\hline CGGA_474 & TCGA-HW-7493-01 \\
\hline CGGA_507 & TCGA-E1-5303-01 \\
\hline CGGA_1862 & TCGA-S9-A6WL-01 \\
\hline CGGA_P319 & TCGA-DU-6395-01 \\
\hline CGGA_1886 & TCGA-HT-A614-01 \\
\hline CGGA_1446 & TCGA-HW-7490-01 \\
\hline CGGA_2024 & TCGA-DU-7014-01 \\
\hline CGGA_P172 & TCGA-E1-5302-01 \\
\hline CGGA_1610 & TCGA-TM-A84F-01 \\
\hline CGGA_28 & TCGA-TQ-A8XE-02 \\
\hline CGGA_831 & TCGA-CS-6290-01 \\
\hline CGGA_1699 & TCGA-HT-A616-01 \\
\hline CGGA_720 & TCGA-FG-A4MT-01 \\
\hline CGGA_1391 & TCGA-HT-7686-01 \\
\hline CGGA_P87 & TCGA-DB-A64W-01 \\
\hline CGGA_P113 & TCGA-FG-A713-01 \\
\hline CGGA_1670 & TCGA-S9-A7R4-01 \\
\hline CGGA_1141 & TCGA-DB-5278-01 \\
\hline CGGA_1544 & TCGA-HT-7476-01 \\
\hline CGGA_1429 & TCGA-DU-6393-01 \\
\hline CGGA_1130 & TCGA-F6-A8O4-01 \\
\hline CGGA_P609 & TCGA-HT-7902-01 \\
\hline CGGA_883 & TCGA-DU-7299-01 \\
\hline CGGA_420 & TCGA-DU-5872-01 \\
\hline
\end{tabular}

\begin{tabular}{|c|c|}
\hline Symbol & Symbol \\
\hline CGGA_P306 & TCGA-FG-7638-01 \\
\hline CGGA_1457 & TCGA-HW-7489-01 \\
\hline CGGA_1553 & TCGA-DB-A4XH-01 \\
\hline CGGA_1491 & TCGA-HT-8018-01 \\
\hline CGGA_568 & TCGA-HT-7676-01 \\
\hline CGGA_1548 & TCGA-DB-A75P-01 \\
\hline CGGA_1770 & TCGA-DH-5142-01 \\
\hline CGGA_1694 & TCGA-P5-A72W-01 \\
\hline CGGA_P164 & TCGA-DU-7294-01 \\
\hline CGGA_1743 & TCGA-HT-7606-01 \\
\hline CGGA_1607 & TCGA-DU-8162-01 \\
\hline CGGA_358 & TCGA-DU-5853-01 \\
\hline CGGA_1815 & TCGA-DU-A6S3-01 \\
\hline CGGA_1870 & TCGA-TQ-A7RK-01 \\
\hline CGGA_1624 & TCGA-QH-A6X5-01 \\
\hline CGGA_1558 & TCGA-DU-6403-01 \\
\hline CGGA_487 & TCGA-FG-A4MT-02 \\
\hline CGGA_P100 & TCGA-CS-5396-01 \\
\hline CGGA_1454 & TCGA-HT-7479-01 \\
\hline CGGA_1702 & TCGA-DH-A7UU-01 \\
\hline CGGA_825 & TCGA-HT-8563-01 \\
\hline CGGA_2115 & TCGA-P5-A735-01 \\
\hline CGGA_P22 & TCGA-HT-7691-01 \\
\hline CGGA_P461 & TCGA-HT-7680-01 \\
\hline CGGA_406 & TCGA-RY-A83Z-01 \\
\hline CGGA_1228 & TCGA-HT-7603-01 \\
\hline CGGA_1537 & TCGA-DU-7010-01 \\
\hline CGGA_1390 & TCGA-DB-A75O-01 \\
\hline CGGA_1359 & TCGA-TM-A7C5-01 \\
\hline CGGA_1462 & TCGA-HT-7467-01 \\
\hline CGGA_1619 & TCGA-P5-A733-01 \\
\hline CGGA_2003 & TCGA-HW-A5KM-01 \\
\hline CGGA_1459 & TCGA-FG-7643-01 \\
\hline CGGA_1437 & TCGA-HT-A74O-01 \\
\hline CGGA_1745 & TCGA-HT-7485-01 \\
\hline CGGA_1588 & TCGA-DU-6394-01 \\
\hline CGGA_1615 & TCGA-FG-A60J-01 \\
\hline CGGA_1817 & TCGA-FG-A60L-01 \\
\hline CGGA_1500 & TCGA-S9-A6TU-01 \\
\hline CGGA_1538 & TCGA-FG-A711-01 \\
\hline CGGA_2062 & TCGA-HT-7608-01 \\
\hline CGGA_1908 & TCGA-CS-6188-01 \\
\hline CGGA_1698 & TCGA-S9-A6WG-01 \\
\hline CGGA_139 & TCGA-FG-A6J1-01 \\
\hline CGGA_1469 & TCGA-CS-4938-01 \\
\hline CGGA_1521 & TCGA-CS-4942-01 \\
\hline CGGA_1474 & TCGA-HT-7687-01 \\
\hline CGGA_1424 & TCGA-P5-A5EY-01 \\
\hline CGGA_1729 & TCGA-HW-7486-01 \\
\hline
\end{tabular}


Supplementary table S1. The sample ID of TCGA and CGGA (continued)

\begin{tabular}{|c|c|}
\hline Symbol & Symbol \\
\hline CGGA_1529 & TCGA-DU-8167-01 \\
\hline CGGA_777 & TCGA-WH-A86K-01 \\
\hline CGGA_1865 & TCGA-DH-5144-01 \\
\hline CGGA_P308 & TCGA-HT-7471-01 \\
\hline CGGA_1829 & TCGA-S9-A7J0-01 \\
\hline CGGA_1708 & TCGA-QH-A6X3-01 \\
\hline CGGA_1382 & TCGA-HT-8015-01 \\
\hline CGGA_846 & TCGA-DU-6399-01 \\
\hline CGGA_763 & TCGA-CS-6668-01 \\
\hline CGGA_1326 & TCGA-E1-A7Z2-01 \\
\hline CGGA_369 & TCGA-DU-6407-01 \\
\hline CGGA_1631 & TCGA-WY-A85E-01 \\
\hline CGGA_1651 & TCGA-HT-7602-01 \\
\hline CGGA_P280 & TCGA-TM-A7C3-01 \\
\hline CGGA_619 & TCGA-FG-7636-01 \\
\hline CGGA_1520 & TCGA-DU-A5TP-01 \\
\hline CGGA_1758 & TCGA-FG-5964-01 \\
\hline CGGA_887 & TCGA-QH-A65X-01 \\
\hline CGGA_2053 & TCGA-HT-7684-01 \\
\hline CGGA_P338 & TCGA-S9-A89Z-01 \\
\hline CGGA_1809 & TCGA-R8-A6MO-01 \\
\hline CGGA_1172 & TCGA-DU-7306-01 \\
\hline CGGA_1572 & TCGA-E1-A7YN-01 \\
\hline CGGA_1236 & TCGA-DU-A76K-01 \\
\hline CGGA_1713 & TCGA-DU-5872-02 \\
\hline CGGA_1596 & TCGA-P5-A77W-01 \\
\hline CGGA_1418 & TCGA-P5-A736-01 \\
\hline CGGA_P283 & TCGA-DH-5140-01 \\
\hline CGGA_583 & TCGA-DB-5273-01 \\
\hline CGGA_1682 & TCGA-VM-A8CD-01 \\
\hline CGGA_1030 & TCGA-DU-A7TD-01 \\
\hline CGGA_852 & TCGA-S9-A6WP-01 \\
\hline CGGA_1226 & TCGA-DU-A7TB-01 \\
\hline CGGA_P271 & TCGA-DU-7292-01 \\
\hline CGGA_2013 & TCGA-FG-A87Q-01 \\
\hline CGGA_1760 & TCGA-DU-7301-01 \\
\hline CGGA_1518 & TCGA-HT-A5RC-01 \\
\hline CGGA_2121 & TCGA-DU-A6S8-01 \\
\hline CGGA_1002 & TCGA-CS-4941-01 \\
\hline CGGA_863 & TCGA-DU-6404-01 \\
\hline CGGA_1444 & TCGA-FG-A4MU-01 \\
\hline CGGA_1014 & TCGA-DU-8161-01 \\
\hline CGGA_1205 & TCGA-DU-7018-01 \\
\hline
\end{tabular}

\begin{tabular}{|c|c|}
\hline Symbol & Symbol \\
\hline CGGA_1311 & TCGA-FG-5965-02 \\
\hline CGGA_P108 & TCGA-CS-5395-01 \\
\hline CGGA_1010 & TCGA-S9-A7R2-01 \\
\hline CGGA_1791 & TCGA-DU-6397-02 \\
\hline CGGA_1773 & TCGA-DU-6407-02 \\
\hline CGGA_1478 & TCGA-FG-A87N-01 \\
\hline CGGA_1142 & TCGA-S9-A7IX-01 \\
\hline CGGA_1144 & TCGA-TM-A84C-01 \\
\hline CGGA_1603 & TCGA-HT-8110-01 \\
\hline CGGA_1051 & TCGA-S9-A6UA-01 \\
\hline CGGA_1749 & TCGA-HW-A5KK-01 \\
\hline CGGA_2056 & TCGA-S9-A6UB-01 \\
\hline CGGA_1354 & TCGA-HT-7860-01 \\
\hline CGGA_1419 & TCGA-HT-A617-01 \\
\hline CGGA_1911 & TCGA-DU-5854-01 \\
\hline CGGA_1635 & TCGA-DU-6402-01 \\
\hline CGGA_P266 & TCGA-HT-7882-01 \\
\hline CGGA_P158 & TCGA-HT-A74K-01 \\
\hline CGGA_1075 & TCGA-FG-A6IZ-01 \\
\hline CGGA_2106 & TCGA-HT-A619-01 \\
\hline CGGA_1514 & TCGA-DU-6392-01 \\
\hline CGGA_1257 & TCGA-DB-5277-01 \\
\hline CGGA_1430 & TCGA-TM-A84B-01 \\
\hline CGGA_1812 & TCGA-DU-A5TT-01 \\
\hline CGGA_1461 & TCGA-QH-A6CS-01 \\
\hline CGGA_P165 & TCGA-CS-5397-01 \\
\hline CGGA_1100 & TCGA-QH-A6CV-01 \\
\hline CGGA_1380 & TCGA-QH-A6CX-01 \\
\hline CGGA_1681 & TCGA-DU-7012-01 \\
\hline CGGA_1185 & TCGA-DU-A5TY-01 \\
\hline CGGA_1248 & TCGA-DU-6404-02 \\
\hline CGGA_1820 & TCGA-TQ-A7RP-01 \\
\hline CGGA_P159 & TCGA-E1-A7YD-01 \\
\hline CGGA_P104 & TCGA-DU-7013-01 \\
\hline CGGA_1976 & TCGA-HT-A4DS-01 \\
\hline CGGA_1365 & TCGA-HT-8104-01 \\
\hline CGGA_1611 & TCGA-FG-A70Z-01 \\
\hline CGGA_1387 & TCGA-HT-A61C-01 \\
\hline CGGA_1542 & TCGA-VM-A8C9-01 \\
\hline CGGA_1255 & TCGA-KT-A7W1-01 \\
\hline CGGA_1497 & TCGA-TM-A84J-01 \\
\hline CGGA_1137 & TCGA-E1-A7YQ-01 \\
\hline CGGA_1138 & TCGA-HT-8011-01 \\
\hline
\end{tabular}

\begin{tabular}{|c|c|}
\hline Symbol & Symbol \\
\hline CGGA_1476 & TCGA-DU-5874-01 \\
\hline CGGA_1101 & TCGA-DU-8158-01 \\
\hline CGGA_P178 & TCGA-DU-7290-01 \\
\hline CGGA_1198 & TCGA-HT-7857-01 \\
\hline CGGA_1353 & TCGA-DU-A7TJ-01 \\
\hline CGGA_1481 & TCGA-FG-5963-01 \\
\hline CGGA_1807 & TCGA-HT-A5RA-01 \\
\hline CGGA_1262 & TCGA-S9-A6WM-01 \\
\hline CGGA_1378 & TCGA-DU-6410-01 \\
\hline CGGA_P29 & TCGA-DB-A64O-01 \\
\hline CGGA_P25 & TCGA-DH-A7UR-01 \\
\hline CGGA_1666 & TCGA-CS-4943-01 \\
\hline CGGA_P115 & TCGA-DU-6406-01 \\
\hline CGGA_P610 & TCGA-FG-6688-01 \\
\hline CGGA_1120 & TCGA-VW-A8FI-01 \\
\hline CGGA_1696 & TCGA-HT-A74H-01 \\
\hline CGGA_1709 & TCGA-E1-A7YJ-01 \\
\hline CGGA_1503 & TCGA-DU-7006-01 \\
\hline CGGA_1656 & TCGA-HT-7616-01 \\
\hline CGGA_1678 & TCGA-S9-A6U0-01 \\
\hline CGGA_P175 & TCGA-FG-A4MW-01 \\
\hline CGGA_P136 & TCGA-DU-5847-01 \\
\hline CGGA_P116 & TCGA-E1-A7YL-01 \\
\hline CGGA_120 & TCGA-HT-A5R9-01 \\
\hline CGGA_1086 & TCGA-TQ-A7RM-01 \\
\hline CGGA_1422 & TCGA-DU-6405-01 \\
\hline CGGA_1445 & TCGA-DB-5274-01 \\
\hline CGGA_P3 & TCGA-FG-5963-02 \\
\hline CGGA_1494 & TCGA-QH-A6XC-01 \\
\hline CGGA_1780 & TCGA-DU-A7T6-01 \\
\hline CGGA_1722 & TCGA-P5-A72Z-01 \\
\hline CGGA_1282 & TCGA-CS-6186-01 \\
\hline CGGA_P265 & TCGA-DU-5852-01 \\
\hline CGGA_P23 & TCGA-P5-A72U-01 \\
\hline CGGA_1767 & TCGA-E1-A7YI-01 \\
\hline CGGA_P180 & TCGA-S9-A7IS-01 \\
\hline CGGA_1486 & TCGA-DU-8165-01 \\
\hline CGGA_P205 & TCGA-DU-A76L-01 \\
\hline \multirow[t]{5}{*}{ CGGA_1564 } & TCGA-DU-7304-02 \\
\hline & TCGA-DH-A669-01 \\
\hline & TCGA-FG-6692-01 \\
\hline & TCGA-FG-A6J3-01 \\
\hline & TCGA-DH-A669-02 \\
\hline
\end{tabular}


Supplementary table S2. PCA result

\begin{tabular}{llcccccccccc}
\hline & PC1 & PC2 & PC3 & PC4 & PC5 & PC6 & PC7 & PC8 & PC9 & PC10 \\
\hline \multirow{4}{*}{ CGGA } & Standard deviation & 3.1428 & 0.24215 & 0.13171 & 0.11793 & 0.10335 & 0.08359 & 0.08156 & 0.06177 & 0.05141 & 0.04711 \\
\cline { 2 - 11 } & Proportion of Variance & 0.9877 & 0.00586 & 0.00173 & 0.00139 & 0.00107 & 0.00070 & 0.00067 & 0.00038 & 0.00026 & 0.00022 \\
\cline { 2 - 11 } & Cumulative Proportion & 0.9877 & 0.99357 & 0.99531 & 0.99670 & 0.99777 & 0.99847 & 0.99913 & 0.99951 & 0.99978 & 1.00000 \\
\hline \multirow{2}{*}{ TCGA } & Standard deviation & 1.7676 & 1.4681 & 1.0854 & 0.91227 & 0.89266 & 0.7497 & 0.67143 & 0.6115 & 0.53706 & 0.48771 \\
\cline { 2 - 10 } & Proportion of Variance & 0.3125 & 0.2155 & 0.1178 & 0.08322 & 0.07968 & 0.0562 & 0.04508 & 0.0374 & 0.02884 & 0.02379 \\
\cline { 2 - 10 } & Cumulative Proportion & 0.8125 & 0.8280 & 0.8458 & 0.82900 & 0.90869 & 0.9149 & 0.92997 & 0.9474 & 0.97621 & 0.02379 \\
\hline
\end{tabular}

